\title{
Modulated Amplitude Waves and Defect Formation in the One-Dimensional Complex Ginzburg-Landau Equation
}

\author{
Lutz Brusch $^{\mathrm{a}}$, Alessandro Torcini ${ }^{\mathrm{b}}$, Martin van Hecke ${ }^{\mathrm{ac}}$, Martín G. Zimmermann ${ }^{\mathrm{d}}$ and Markus \\ Bära \\ a Max-Planck-Institut für Physik komplexer Systeme, Nöthnitzer Straße 38, D-01187 Dresden, \\ Germany \\ b Dipartimento di Fisica, Universita' "La Sapienza", P.le A. Moro 2, I-00185 Roma, Italy and \\ Istituto Nazionale di Fisica della Materia, Unità di Firenze, Largo Enrico Fermi 2, I-50125 \\ Firenze, Italy \\ ${ }^{\mathrm{c}}$ Center for Chaos and Turbulence Studies, The Niels Bohr Institute, Blegdamsvej 17, 2100 \\ Copenhagen, Denmark and \\ Kamerlingh Onnes Laboratory, Leiden University, Niels Bohrweg 2, 2333CA Leiden, The \\ Netherlands \\ d Instituto Mediterráneo de Estudios Avanzados, IMEDEA (CSIC-UIB), E-07071 Palma de \\ Mallorca, Spain and \\ Departamento de Física, FCEN-Universidad de Buenos Aires, Pab. I Ciudad Universitaria, \\ 1428 Buenos Aires, Argentina
}

\begin{abstract}
The transition from phase chaos to defect chaos in the complex Ginzburg-Landau equation (CGLE) is related to saddle-node bifurcations of modulated amplitude waves (MAWs). First, the spatial period $P$ of MAWs is shown to be limited by a maximum $P_{S N}$ which depends on the CGLE coefficients; MAW-like structures with period larger than $P_{S N}$ evolve to defects. Second, slowly evolving near-MAWs with average phase gradients $\nu \approx 0$ and various periods occur naturally in phase chaotic states of the CGLE. As a measure for these periods, we study the distributions of spacings $p$ between neighboring peaks of the phase gradient. A systematic comparison of $p$ and $P_{S N}$ as a function of coefficients of the CGLE shows that defects are generated at locations where $p$ becomes larger than $P_{S N}$. In other words, MAWs with period $P_{S N}$ represent "critical nuclei" for the formation of defects in phase chaos and may trigger the transition to defect chaos. Since rare events where $p$ becomes sufficiently large to lead to defect formation may only occur after a long transient, the coefficients where the transition to defect chaos seems to occur depend on system size and integration time. We conjecture that in the regime where the maximum period $P_{S N}$ has diverged, phase chaos persists in the thermodynamic limit.
\end{abstract}

PACS: 05.45.Jn 03.40.Kf; 05.45.-a;

Keywords: Phase chaos, Defect chaos, Complex Ginzburg-Landau equation, Coherent structures 


\section{Introduction}

The transition from phase to defect chaos for the one dimensional complex Ginzburg-Landau equation (CGLE) was recently related to the bifurcation properties of a family of coherent structures called modulated amplitude waves (MAWs) [1]. In this paper the relationship between MAWs and large scale chaos is studied in detail, providing a comprehensive description of various aspects of the CGLE chaotic dynamics.

When a spatially extended system is driven sufficiently far away from equilibrium, patterns can eventually form [2,3]. In many cases these patterns show an erratic behavior in space and time: such behavior is commonly referred to as spatiotemporal chaos [2 [5]. Examples of extended systems displaying such chaotic dynamics in one spatial dimension include: heated wire convection [6], printers instability and film drag experiments [7], eutectic growth [8], binary convection [9], sidewall convection [10], the far field of spiral waves in the Belousov-Zhabotinsky reaction [11], the Taylor-Dean system [12], hydrothermal [13] and internal [14] waves excited by the Marangoni effect and the oscillatory instability of a Rayleigh-Bénard convection pattern [15].

Near the pattern forming threshold, the dynamics of such systems can often be described by so-called amplitude equations. When the pattern forming bifurcation from the homogeneous state is a forward Hopf bifurcation, the appropriate amplitude equation is the CGLE [2,3], which in one spatial dimension reads as:

$\partial_{t} A=A+\left(1+i c_{1}\right) \partial_{x}^{2} A-\left(1-i c_{3}\right)|A|^{2} A$

where $c_{1}$ and $c_{3}$ are real coefficients and the field $A=A(x, t)$ has complex values.

For different choices of the coefficients numerical investigations of the CGLE have revealed the existence of various steady and spatiotemporally chaotic states [1 $5,15,26$. Many of these states appear to consist of individual structures with well defined propagation and interaction properties. It is thus tempting to use these structures as building blocks for a better understanding of spatiotemporal chaos. In this paper we will essentially follow such an approach.

As a function of the coefficients $c_{1}$ and $c_{3}$, the CGLE (1) can exhibit two qualitatively different spatiotemporal chaotic states known as phase chaos (when the modulus $|A|$ is at any time bounded away from zero) and defect chaos (when $|A|$ can vanish leading to phase singularities). It is under dispute whether the transition from phase to defect chaos is sharp or not, and if a pure phase-chaotic, (i.e., defect-free) state can persist in the thermodynamic limit [21,27]. We will address these issues by suggesting a mechanism for the formation of defects related to the range of existence of MAWs.

The main points of this paper are outlined in the following and illustrated in Figs. 1, 2. (i) Our investigation starts with the study of MAWs, which are uniformly propagating, spatially periodic solutions of the CGLE. These MAWs are parameterized by the average phase gradient $\nu$ and their spatial period $P$. Our study is confined to the case $\nu=0$ for reasons specified below. Spatial profiles and the stable propagation of a particular MAW are presented in Fig. 11a-c. Isolated MAW structures consisting of just one spatial period $P$ play an important role in defect formation. In particular, for fixed CGLE coefficients the range of existence of coherent MAWs is limited by a saddle-node $(S N)$ bifurcation which occurs when $P$ reaches a maximal period $P_{S N}$. (ii) If the MAWs are driven into conditions with $P>P_{S N}$ a dynamical instability occurs leading to the formation of defects (Fig. 1 $1 \mathrm{~d}$ ). (iii) Slowly evolving structures reminiscent of MAWs ("near-MAWs") are observed in the phase chaotic regime (Fig. 1e,f). In order to characterize such states, we have examined the distribution $D(p)$ of spacings $p$ between neighboring peaks of the phase-gradient profile. In particular for sufficiently long spacing $p$, the observed phase chaos structures are often very similar to a single period of a coherent MAW (Fig. 1 $1 \mathrm{f}$ ). (iv) When a 

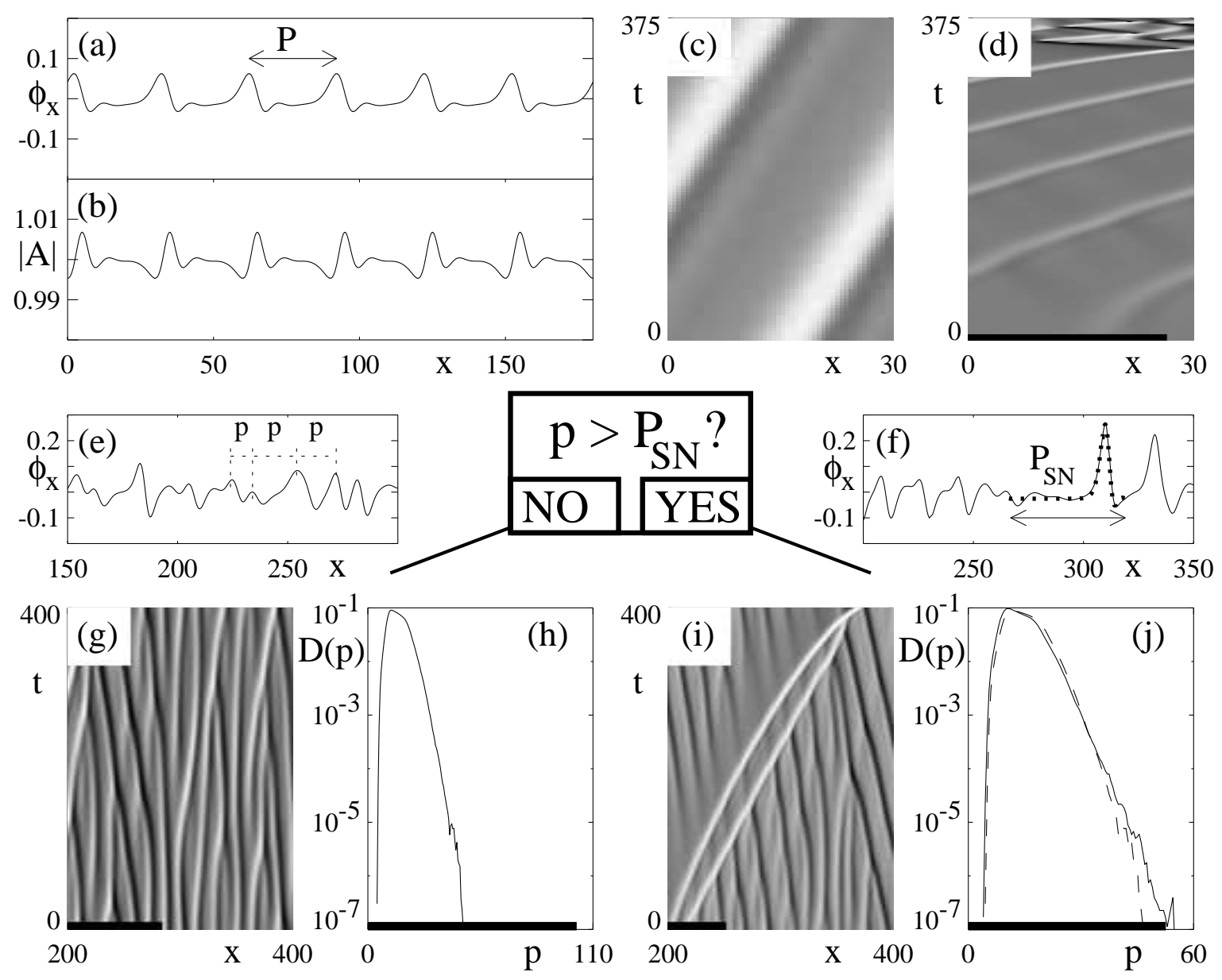

Figure 1. Summary of our main results which constitute a picture for the formation of defects from phase chaotic states. (a,b) Example of a coherent structure: phase gradient and modulus of a period $P=30 \mathrm{MAW}$ at $c_{1}=0.6, c_{3}=2$. (c) Space time plot showing the stable propagation of the MAW from $(\mathrm{a}, \mathrm{b})$ in a small system of size $P$ with periodic boundary conditions. Subsequent space time plots also show the phase gradient encoded in gray-scale (minima appear dark, maxima bright). (d) The same MAW as initial condition creates defects at $c_{1}=0.7, c_{3}=2$ where $P>P_{S N}=26.8$. Black bars above the x-axis denote the size of $P_{S N}$ specific to the parameters of the panel. (e,g,h) Large scale chaos at $c_{1}=0.63, c_{3}=2, L=512$. (e) Snapshot of the phase gradient profile with individual inter-peak spacings $p$. (g) Space time evolution of phase chaos and (h) distribution $D(p)$ showing $p \ll P_{S N}$ and no defects. A transient of $t \approx 10^{4}$ is not shown. (f,i,j) Large scale chaos at $c_{1}=0.65, c_{3}=2, L=512$. (f) Snapshot of the phase gradient profile $t=120$ before the first defect forms and the MAW (dotted, $P=P_{S N}$ ) overlayed onto the long structure. (i) Transient phase chaos with a fast and long structure traveling through the system which eventually nucleates defect chaos at $t=400, x=360$ (a transient of $t \approx 10^{4}$ is not shown). A snapshot of this structure was shown in (f). (j) The tail of the distribution of $p$ reaches $p>P_{S N}$ due to the long structure; this leads to the break down of phase chaos. The distribution $D(p)$ shown in (h) is also reported (dashed line). From the comparison of the two it is evident that the distributions do not modify dramatically when $c_{1}$ is increased, while $P_{S N}$ decreases noticeably. 


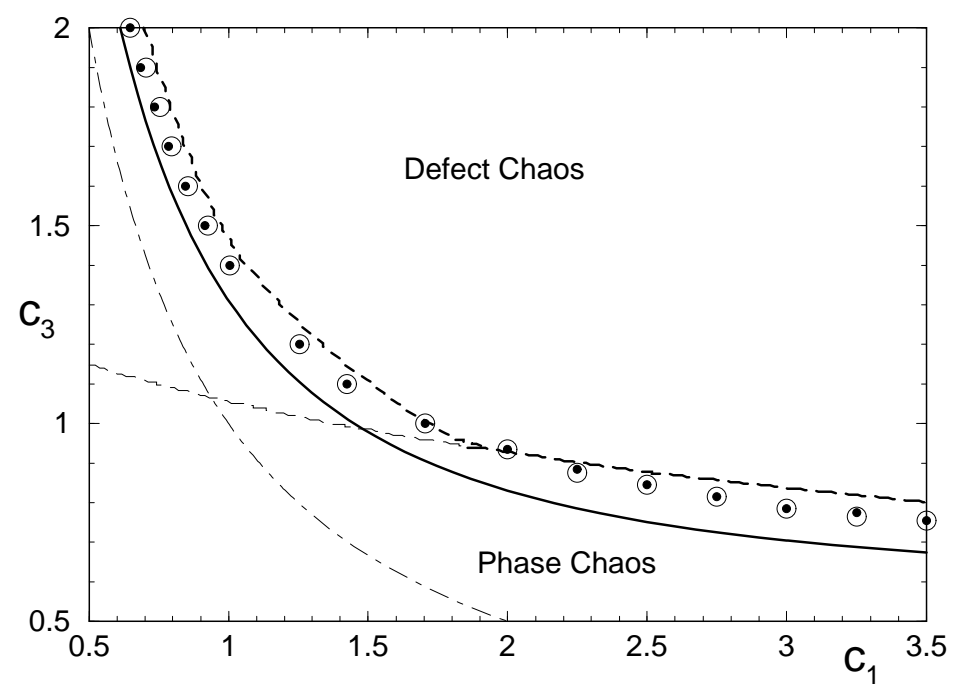

Figure 2. Phase diagram of the CGLE, showing the Benjamin-Feir-Newell curve (thin dotdashed) where the transition from stable homogeneous oscillations to phase chaos takes place. The curves $L_{1}$ (long dashed), $L_{2}$ (thin dashed) and $L_{3}$ (dashed) as obtained in [17, 19] separate the various chaotic states. The filled circles correspond to our estimates of the $L_{1}$ and $L_{3}$ transitions based on direct simulations of the CGLE along the 17 cuts in coefficient space that we studied. The open circles correspond to the location in coefficient space where the maximal inter-peak spacing $p_{\max }$ is equal to the maximal MAW period $P_{S N}$. Only small discrepancies between these two can be observed. Finally the full curve shows the $P_{S N} \rightarrow \infty$ limit which we conjecture to be a lower boundary for the transition from phase to defect chaos.

phase chaotic state displays spacings $p$ larger than $P_{S N}$, phase chaos breaks down and defects are formed (e.g. at $t=400, x=360$ in Fig. 1 i i). Thus, the MAW with $P=P_{S N}$ may be viewed as a "critical nucleus" for the creation of defects. In phase chaos defect formation is similar to the dynamical process by which isolated MAW structures generate defects (Fig. 11). Therefore purely phase chaotic states are those for which $p$ remains bounded below $P_{S N}$ (Fig. 1 $1 \mathrm{~g}$ ), while defect chaos can occur when $p$ becomes larger than $P_{S N}$ (Fig. 1ii). ( $v$ ) A more detailed study of the probability distribution of the $p$ 's shows that for large $p$ the probability decays exponentially (Fig. 1 $1 \mathrm{~h}, \mathrm{j}$ ). As long as $P_{S N}$ has a finite value, we expect that, possibly after a very long transient time, defects will be generated. (vi) However, in a finite domain of the phase chaotic region, MAWs of arbitrarily large $P$ exist: we expect that in this region, even in the thermodynamic limit, phase chaos will persist. Fig. 2 shows the main quadrant of the CGLE coefficient space. The region of persistent phase chaos is bounded by the Benjamin-Feir-Newell curve (thin dotdashed) and the curve along which $P_{S N} \rightarrow \infty$ (full curve in Fig. 2).

The outline of this paper is as follows: Section 2 is devoted to the study of the coherent MAW structures. In section 2.2 we study the bifurcation diagram of the MAWs, starting from the homogeneous oscillation. In section 2.3 the incoherent dynamics of near-MAW structures is presented. We show that for $p>P_{S N}$, i.e., beyond the saddle-node bifurcation, near-MAWs evolve to defects. To illustrate the origin of the saddle-node bifurcations in section 2.4 we compare bifurcation diagrams of coherent structures for different phase gradient expansions of the CGLE. For the lowest order expansion (known as the Kuramoto-Sivashinsky equation [2]) the saddle-node bifurcation is absent while it is captured by expansions of higher order. This explains why the divergence of the phase gradient was exclusively observed in simulations [20] 
of higher order expansions. In section 3 we study various aspects of spatiotemporal chaos in the CGLE, and relate the observed continuous $\left(L_{1}\right)$ and discontinuous $\left(L_{3}\right)$ transitions (see Fig. 2) to properties of the MAWs. The transition to defect chaos takes place when near-MAWs with periods larger than $P_{S N}$ occur in a phase chaotic state. In section 3.4 the typical values of $p$ in the phase chaotic regime are related to the competition of two instabilities of the MAWs, and it is possible to give a good estimate for the numerically measured transition from phase to defect chaos from these considerations. A discussion of the presented results and some final remarks are reported in section 1 .

\section{Modulated Amplitude Waves}

In this section we study the main properties of modulated amplitude waves (MAWs) [1]. First, in section 2.1 the coherent structure framework that we use to describe the MAWs is introduced. The bifurcation diagram of MAWs is explored in section 2.2, with a particular focus on the saddle-node bifurcations that limit the range of existence of MAWs. In section 2.3 we study the nonlinear evolution of near-MAWs that are "pushed" beyond their saddlenode bifurcation and show that this leads to the formation of defects. Finally, in section 2.4 a bifurcation analysis of MAW-like coherent structures is performed in various phase equations that have been proposed as approximated models for the phase chaotic dynamics of the CGLE, and we show that only higher order phase equations reproduce the saddle-node bifurcation.
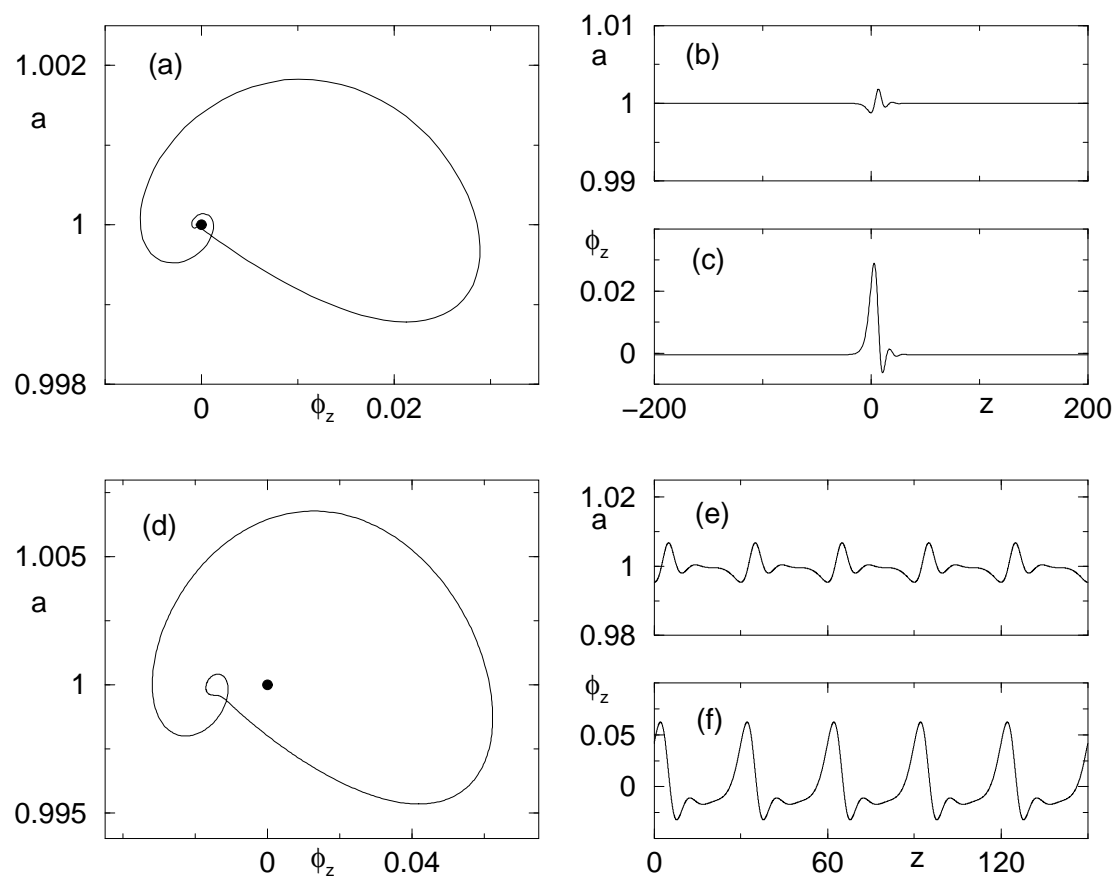

Figure 3. Examples of ODE solutions and corresponding amplitude and phase gradient profiles of MAWs. (a) Homoclinic orbit for $c_{1}=0.55$ and $c_{3}=2$; (b,c) corresponding profiles. (d) Limit cycle for $c_{1}=0.60, c_{3}=2$ and $P=30 ;(\mathrm{e}, \mathrm{f})$ corresponding profiles. Dots in $(\mathrm{a}, \mathrm{d})$ denote the unstable fixed point $(1,0,0)$ from which these orbits emerged. 


\subsection{Coherent Structures}

Coherent structures in the CGLE are uniformly propagating structures of the form

$A(x, t)=a(x-v t) e^{i \phi(x-v t)} e^{i \omega t}$,

where $a$ and $\phi$ are real-valued functions of $z:=x-v t$. Coherent structures have been studied extensively [23 25] and play an important role in various regimes of the CGLE [16,22, 25].

The restriction to uniformly propagating structures reduces the CGLE to a set of three coupled ordinary differential equations (ODEs) [28]. These ODEs are readily found by substitution of Ansatz (2) into the CGLE (11) and read as:

$\begin{aligned} a_{z} & =b \\ b_{z} & =\psi^{2} a-\gamma^{-1}\left[\left(1-c_{1} \omega\right) a+v\left(b+c_{1} \psi a\right)-\left(1-c_{1} c_{3}\right) a^{3}\right] \\ \psi_{z} & =-2 b \psi / a+\gamma^{-1}\left[c_{1}+\omega+v\left(c_{1} b / a-\psi\right)-\left(c_{1}+c_{3}\right) a^{2}\right],\end{aligned}$

where $b:=a_{z}, \psi:=\phi_{z}$, and $\gamma:=1+c_{1}^{2}$. Solutions of the ODEs (3) correspond to coherent structures of the CGLE.

The simplest relevant solutions of these ODEs are the fixed points given by $(a, b, \psi)=$ $\left(\sqrt{1-q^{2}}, 0, q\right)$; these correspond to plane wave solutions of the CGLE where $A(x, t)=\sqrt{1-q^{2}} \exp i(q x+\omega t)$ and $\omega=c_{3}-q^{2}\left(c_{1}+c_{3}\right)$. An example of more complex solutions of the ODEs (3) are heteroclinic orbits which correspond to coherent structures that asymptotically connect different states. Examples of such structures are fronts that connect nonlinear plane waves to the homogeneous state $A=0$ [24 and Nozaki-Bekki holes that connect plane waves of different wavenumber $q$ [24,29.

Here we present an extensive study of the structures that are associated with the limit cycles of the ODEs (3) [30]. These limit cycles correspond to spatially periodic solutions of the CGLE that we have already referred to as MAWs (Fig. 3). For appropriate choices of $c_{1}$ and $c_{3}$, the period $P$ of these MAWs can be made arbitrarily large, and in this limit the limit cycles approach a homoclinic orbit connecting the stable and unstable manifold of one of the plane wave fixed points (Fig. 3a). Some of these infinite period MAWs have also been referred to as "homoclinic" holes, and have been studied extensively recently [25,31,; they are qualitatively different from the well-known Nozaki-Bekki holes [29].

Even if the coefficients $c_{1}$ and $c_{3}$ are fixed, MAWs are not uniquely determined. Counting arguments, similar to those developed in [24], yield that in general we may expect a two-parameter family of solutions. Let us first perform the counting for the homoclinic orbits. As shown in [25], these orbits connect the one-dimensional unstable manifold of a fixed point with its twodimensional stable manifold. In general, one needs to satisfy one condition to make such a connection, in other words, such a homoclinic orbit is of codimension one. Since the coherent structure Ansatz (2) has two freely adjustable parameters ( $\omega$ and $v$ ), we therefore expect a one parameter family of homoclinic orbits.

The situation for the limit cycles of the ODEs is even simpler. Limit cycles are of codimension zero in parameter space, and so we expect a two parameter family of limit cycles. In other words, if we have found a limit cycle for certain values of $v$ and $\omega$, then we expect this limit cycle to persist for nearby values of the parameters $v$ and $\omega$.

Obviously, we can parameterize this family of limit cycle coherent structures by $v$ and $\omega$, but this is not very insightful. Instead we will use the following two quantities that are more directly accessible in studies of the CGLE: the spatial period $P$ of the MAWs, and their average phase gradient $\nu:=\left(\int_{0}^{P} d x \phi_{x}\right) / P$. Note that for homoclinic holes, $P$ simply goes to infinity; thus homoclinic orbits and limit cycles are members of a single family. 
The multiplicity of the MAWs can also be obtained by considering the instability of the plane wave solutions from which the MAWs emerge [32] (see section 2.2 below). The plane waves form a one-parameter $(q)$ family and undergo the well-known Eckhaus instability when the coefficients $c_{1}, c_{3}$ are increased beyond certain critical values which depend on $q$. In the unstable regime, a plane wave with wavenumber $q$ is unstable to a whole band of perturbations with wavenumbers $k \in\left[0, k_{\max }(q)\right]$ 画. For finite systems of size $L$, this instability thus only appears when $L>L_{\min }=2 \pi / k_{\max }$. Therefore for each $q$ there is a unique one-parameter $(L)$ family of perturbations that can render the plane wave unstable and at each of the corresponding bifurcations a new MAW solution emerges. Hence also by this line of reasoning MAWs form a two-parameter family.

\subsection{Bifurcation Scenario for MAWs}
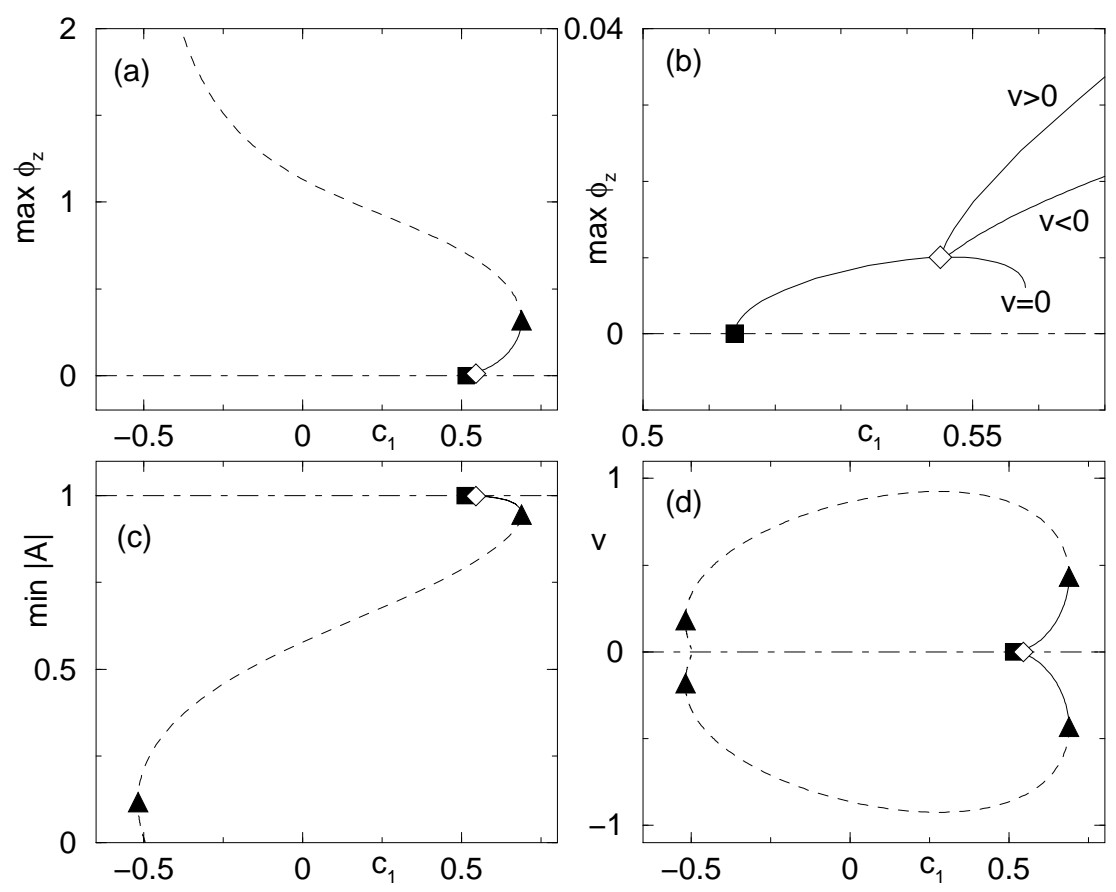

Figure 4. Bifurcation diagrams for fixed $c_{3}=2$ and $P=30$, showing Hopf (filled square), drift pitchfork (open diamond) and saddle-node (triangle) bifurcations. The dot-dashed line represents the homogeneously oscillating solution of the CGLE, while lower and upper branch MAWs are represented by full and dashed curves respectively. (a) Overview of the maximum phase gradient of the MAWs as function of $c_{1}$, (b) close-up, (c) the minimum of $|A|$, and (d) the velocity $v$. For details see text.

The general counting arguments given in the previous section do not provide information on the range of existence of MAWs as a function of the coefficients $c_{1}$ and $c_{3}$ and the parameters $\nu$ and $P$. Here we will focus our analysis on the $\nu=0$ case since this is most relevant for the transition to defect chaos [33; the $\nu \neq 0$ case will be treated elsewhere [34.

All bifurcation computations have been performed with the aid of the software package AUTO94 [35]. AUTO94 can trace MAW solutions through parameter space, and when it detects bifurcations it can follow the newly emerging branches. AUTO94 discretizes the ODEs (3) on 

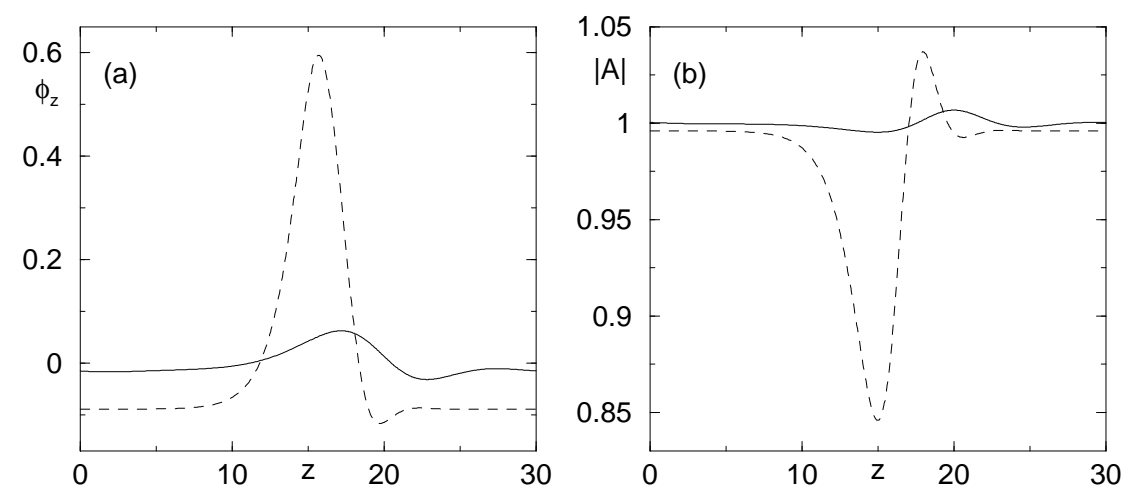

Figure 5. (a) Phase gradient and (b) amplitude profiles of a lower branch (full curve) and upper branch (dashed curve) MAW, obtained for $c_{1}=0.6, c_{3}=2, P=30$.

a periodic domain of length $L$, and $L$ will play the role of the period $P$ of the MAWs. Control of the average phase gradient $\nu=\nu_{0}$ is implemented via the integral constraint $\int_{0}^{L} \psi d z=L \nu_{0}$. Since periodic boundary conditions result in translational invariance, we introduce an additional "pinning" condition $a_{z}(0)=0$ in order to obtain unique solutions.

Under these conditions, the continuation procedure works as follows. First of all, $\nu$ and $P$ are set to fixed values, and throughout this paper we will set $\nu=0$. Starting from a known solution such as a plane wave or a coherent structure obtained by other means, AUTO94 is set up to trace the MAWs along trajectories in $c_{1}, c_{3}$ space, while calculating the parameters $\omega$ and $v$ of these MAWs.

The results of our bifurcation analysis are summarized in Fig. 1 . When $c_{1}$ or $c_{3}$ is increased, the uniformly oscillating state of the CGLE $\left(A(x, t)=e^{i c_{3} t}\right)$ becomes unstable via a Hopf bifurcation, from which stationary MAWs emerge (section 2.2.1). These stationary, left-right symmetric solutions undergo a drift pitchfork bifurcation, which leads to left and right traveling MAWs (section 2.2.2, see also Fig. 四); as discussed later, these are the solutions relevant for the dynamics in the phase chaotic regime. Following these branches of traveling MAWs, we encounter a saddle-node bifurcation where an "upper" and "lower" branch of MAWs merge (section 2.2.3, see also Fig. 5); this bifurcation limits the range of existence of MAWs and is closely related to the formation of defects. The upper branch MAWs can be continued back to negative values of $c_{1}$, where they terminate in a solution consisting of a periodic array of shocks and stationary Nozaki-Bekki holes [29]. Upper branch MAWs with $P \rightarrow \infty$ have been studied under the name homoclinic holes 25, 31.

It should be noted that, without loss of generality, we focus here on solutions with $v>0$, for which the main peak of the phase gradient profile is positive (see Fig.5). Solutions with $v<0$ can be obtained from right moving MAWs by applying the mapping $x \rightarrow-x, z \rightarrow-z, v \rightarrow$ $-v, a_{z} \rightarrow-a_{z}, \phi_{z} \rightarrow-\phi_{z}$.

\subsubsection{Benjamin-Feir instability - Hopf bifurcation}

Since the average phase gradient $\nu$ is conserved across bifurcations, we start the continuation procedure from the uniformly oscillating solution $A(x, t)=e^{i c_{3} t}$ that has $\nu=0$. On an infinite domain this uniformly oscillating solution becomes unstable via the so-called Benjamin-Feir instability when $c_{1} c_{3} \geq 1$ [3]. In a finite domain of size $L$, the onset of this instability is shifted to higher values of the product $c_{1} c_{3}$ [36]; this finite size effect is relevant for our studies since the spatial period $L=P$ is fixed in the continuation procedure. 

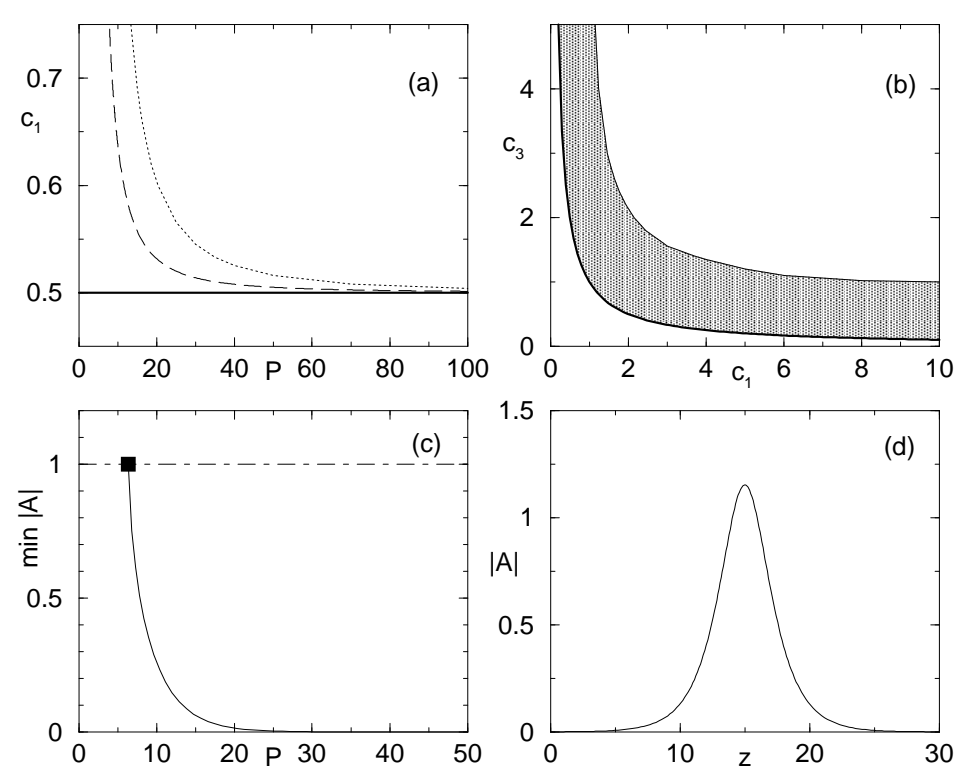

Figure 6. (a) Location of Hopf (dashed curve) and drift pitchfork bifurcation (dotted curve) in $c_{1}, P$ space for $c_{3}=2.0$. (b) The shaded area reported in the $c_{1}, c_{3}$ coefficient space indicates where the drift pitchfork bifurcation does occur. The thick full curve in $(a, b)$ indicates the Benjamin-Feir-Newell instability for infinite domains. (c) Example of a bifurcation diagram for large values of the coefficients $c_{1}=10, c_{3}=5$ where the drift pitchfork bifurcation does not occur. For increasing $P$ the MAW solutions approach regular arrays of stationary pulses; an example of such a pulse is shown in (d) for $P=30$.

In the ODEs (3), the fixed point $(a, b, \psi)=(1,0,0)$ corresponds to the homogeneously oscillating solution. For given values of the period $P$, this fixed point undergoes a Hopf bifurcation at values of $c_{1}$ and $c_{3}$ where in the CGLE (1) the mode with wavenumber $2 \pi / P$ becomes unstable [36]. This Hopf bifurcation was analytically shown to be supercritical for sufficiently small $\nu$ and large $P$ in earlier studies [15.32]; our numerical results are consistent with this. For finite $P$, the solution bifurcating from the fixed point is a limit cycle which approaches a homoclinic orbit in the limit $P \rightarrow \infty$. The solutions of the CGLE that correspond to these orbits are stationary, reflection symmetric MAWs; an example of these is shown in Fig. 9a.

\subsubsection{Drift pitchfork bifurcation}

When the CGLE coefficients $c_{1}$ and/or $c_{3}$ are increased further, the stationary MAW undergoes a drift pitchfork bifurcation [37] from which two new branches of asymmetric $(v \neq 0)$ MAWs emerge (see Fig. $\$ \mathrm{~b}$ ); one of these moves to the left, one to the right. The locations of both the Hopf and the drift pitchfork bifurcation approach the Benjamin-Feir-Newell curve for large $P$ (Fig. 6a), while for smaller $P$ the drift pitchfork occurs for increasingly larger coefficients $c_{1}$ and $c_{3}$. However, only when these coefficient lie in the range shown as the shaded area in Fig. 6b, the pitchfork bifurcation can occur. Otherwise, only stationary MAWs are found. For increasing $c_{1}$ and $c_{3}$ these MAWs become pulse-like and finally approach the solitonic solutions of the nonlinear Schrödinger equation [24] (Fig. 6c,d).

For the case $\nu \neq 0$ [34], the initial plane wave already breaks the reflection symmetry, the initial MAW has nonzero velocity and the drift pitchfork bifurcation is replaced by its typical unfolding [38. 

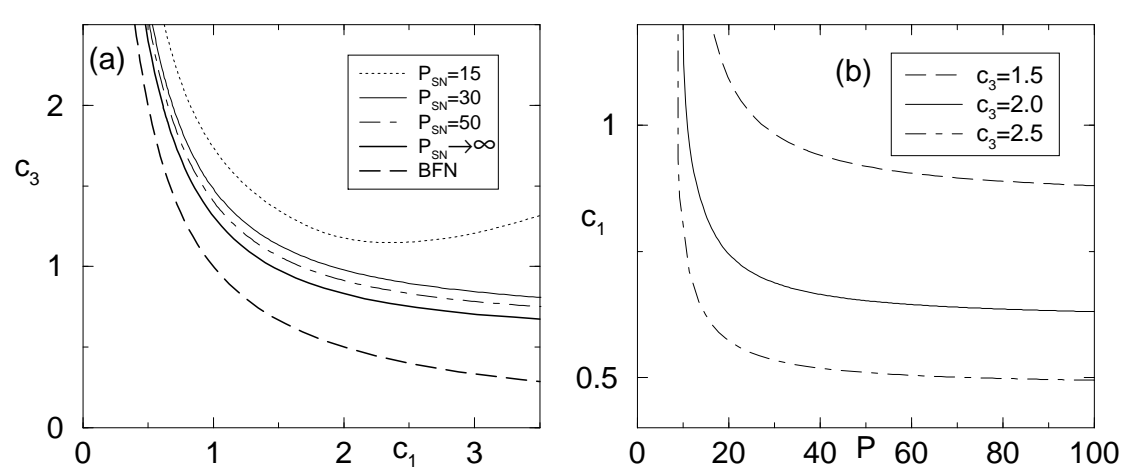

Figure 7. Locations of the saddle-node bifurcations in the $c_{1}, c_{3}$ plane (a) and the $P, c_{1}$ plane (b). BFN denotes the Benjamin-Feir-Newell curve.

\subsubsection{Saddle-node bifurcation}

Along the branch of right traveling MAWs that we described above, the maximum of the phase gradient grows with increasing $c_{1}$ and $c_{3}$ until a saddle-node (SN) bifurcation is reached, where these MAWs merge with another branch of MAW-like solutions. To distinguish these branches we refer to them as the "lower" and the "upper" branch; for examples see Figs. 1, 8 . The lower branch MAWs are the key to understand more of the phenomenology of phase chaos. The upper branch MAWs can, similarly to the lower branch MAWs, be parameterized by $\nu$ and $P$, but for the same parameters, they present more pronounced modulations (see Fig. 5).

The most important aspect of the saddle-node bifurcation is that it limits the range of existence of MAWs, since we will show that this limit is responsible for the transition from phase to defect chaos. Fixing $\nu=0$, the locations of these bifurcations form a two-dimensional manifold in the three dimensional space spanned by $c_{1}, c_{3}$ and $P$. In Fig. 7 a the saddle-node curves are shown in the $c_{1}, c_{3}$ coefficient plane for a number of fixed periods $P$; for larger $P$, the values of $c_{1}, c_{3}$ where the bifurcation takes place decrease. In Fig. $7 \mathrm{~b}$ the saddle-node curves for a number of fixed values of $c_{3}$ are shown in the $P, c_{1}$ plane; for larger $c_{3}\left(c_{1}\right)$, the saddle-node occurs for smaller values of $P$ and $c_{1}\left(c_{3}\right)$ [39]. Once the coefficients $c_{1}$ and $c_{3}$ are fixed, we define $P_{S N}$ as the period for which the saddle-node bifurcation occurs. Note that there is also a range of coefficients $c_{1}$ and $c_{3}$ (between the $P \rightarrow \infty$ and $c_{1} c_{3}=1$ curve where the saddle-node bifurcation does not occur.

\subsection{Evolution of perturbed MAWs}

In this section we will show that many basic aspects of the phenomenology of the CGLE can be understood from a typical bifurcation diagram of MAWs such as shown in Fig. 8. We have chosen fixed coefficients $c_{1}=0.65$ and $c_{3}=2$ and varied the spatial period $P$ of MAWs that exist at these coefficients. Three families of solutions are represented: the homogeneous oscillation, the lower branch (LB) and the upper branch (UB) MAWs. The shaded area schematically indicates the near-MAW structures observed in phase chaotic states such as shown in Fig. 1 (f,i,j). The arrows in Fig. \& represent the dynamical evolution of perturbed MAWs, and their direction can be obtained by performing a linear stability analysis.

Linear stability - As discussed in section 2.2, the homogeneous solution is stable against short wavelength perturbations (arrow 1), and turns unstable via the Hopf bifurcation that also generates the lower branch MAWs (arrows 2). As discussed in [25,31, upper branch MAWs have at least one unstable eigenvalue, and the dynamical evolution of perturbations is directed away from upper branch MAWs (arrows 3,4). 


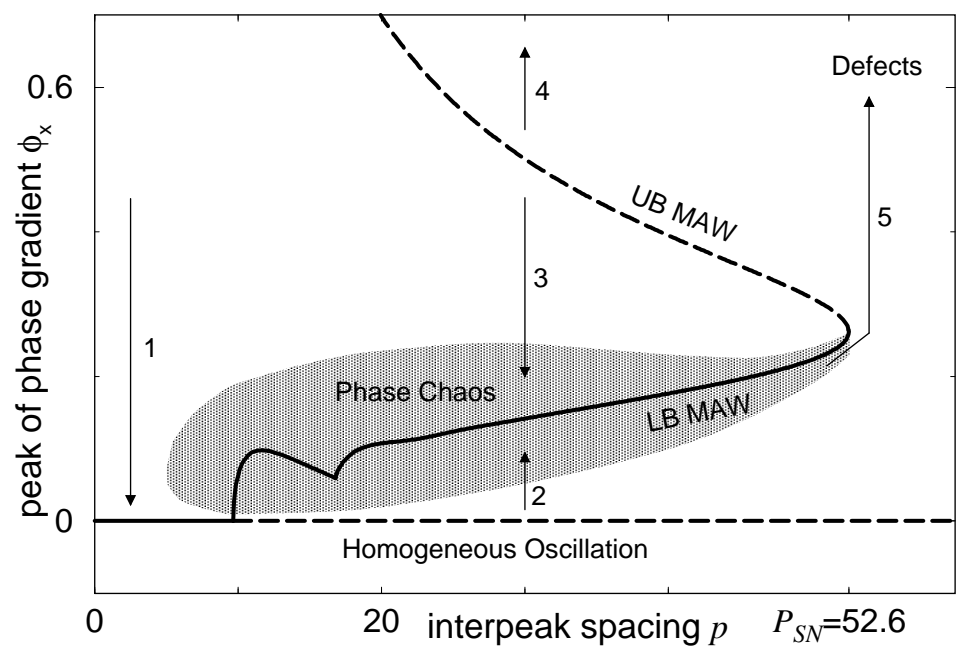

Figure 8. Illustration of the relation between MAW structures and phase chaos for $c_{1}=0.65, c_{3}=$ 2. Both for MAWs and for an extended profile obtained from a phase chaotic state one can extract the values of subsequent phase gradient peaks (vertical axis) and their inter-peak spacing (horizontal axis). The curves show the bifurcation diagram for the lower branch (LB) MAWs and upper branch (UB) MAWs while the shaded area indicates the typical values for near-MAW structures that occur in phase chaos. Full (dashed) curves denote stable (unstable) solutions for system size $L=p$. Arrows show the typical evolutions of near-MAWs. The coefficients here are equal to those in Fig. 1 $(\mathrm{f}, \mathrm{i}, \mathrm{j})$ and for this case we have found that phase chaos is only a long lived transient: the shaded area reaches $P_{S N}$ just before defects appear.

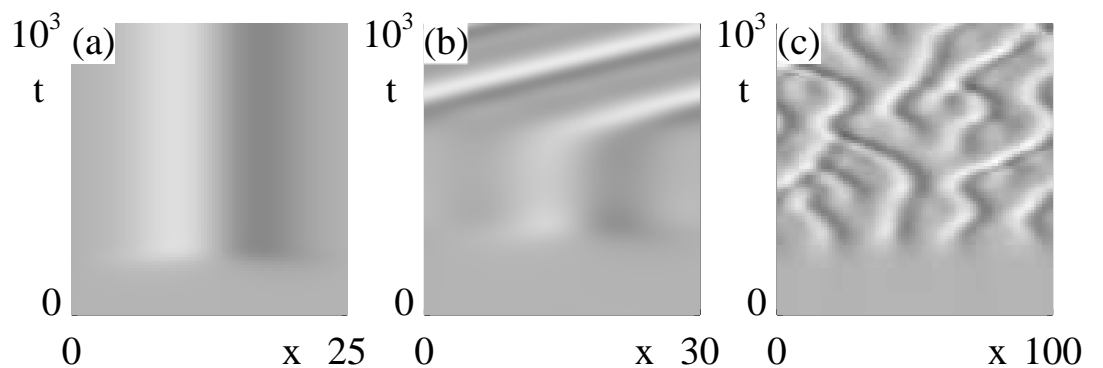

Figure 9. The evolution of an unstable homogeneous state towards lower branch MAW dynamics, for $c_{1}=3$ and $c_{3}=0.6$. The coefficients $c_{1}$ and $c_{3}$ are chosen such that no saddle-node bifurcation occurs for any $P$. (a) Evolution towards a stable stationary lower branch MAW for system size $L=25$ and (b) towards a stable drifting lower branch MAW for system size $L=30$. Note that for the coefficients chosen, the drift pitchfork bifurcation occurs at $P=27.7$. (c) Evolution towards phase chaos for system size $L=100$. Incoherent evolution of structures characterized by local concentrations of phase gradients can be clearly observed. We think of these structures as "near" MAWs. 

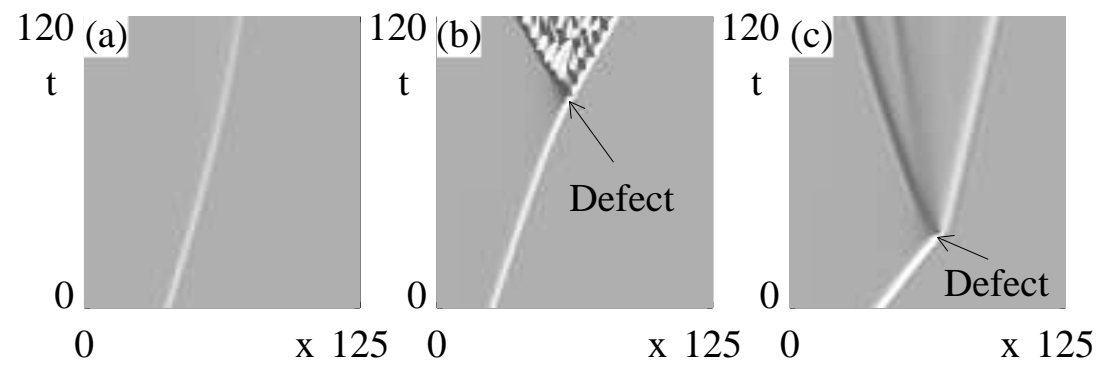

Figure 10. Evolution of perturbations of the upper branch MAWs. The coefficients $c_{1}$ and $c_{3}$ are chosen such that no saddle-node bifurcation occurs for any value of $P$. (a) A slowing down and spreading of the phase gradient characterizes the decay to a lower branch MAW for $c_{1}=0.55$ and $c_{3}=2$. (b) For the same coefficients, another perturbation leads to an increase in velocity and divergence of the phase gradient. A defect occurs, from which hole-defect dynamics spreads for these coefficients. (c) For $c_{1}=3$ and $c_{3}=0.6$ a perturbed upper branch MAW leads to a defect, but defects do not percolate through the system.

The linear stability of lower branch MAWs will be discussed in more detail in section 3.4. It turns out that perturbations of lower branch MAWs can evolve in many ways, but in almost all cases the ensuing dynamics remains close to the lower MAW branch (shaded area in Fig. 8). The only exception we have found to this rule is when a MAW is pushed beyond the saddle-node bifurcation (arrow 5).

Nonlinear evolution - Here we want to go beyond the linear analysis and study the nonlinear evolution of MAWs along the arrows of Fig. 8. The examples (at different choices of the coefficients) of the dynamics shown below are not exhaustive, but should serve to illustrate typical behavior which appears to be very robust.

arrow 2 - When the uniform oscillation becomes linearly unstable perturbations grow. To the left of the saddle-node, perturbations evolve to dynamics dominated by lower branch MAWs (Fig. 9). For small system sizes, stable MAWs may occur (Fig. 9a,b), while for larger systems periodic sequences of MAWs are unstable with respect to the so-called interaction or splitting instabilities [1,40] that will be discussed in section 3.4. Hence a perturbed unstable homogeneous state typically does not converge to a train of coherent MAWs, but instead evolves to phase chaos (Fig. 9c). In the context of the bifurcation diagram, note that the disordered structures observed in the phase chaotic evolution are quite similar to lower branch MAWs. The shaded area in Fig. 8 represents this "near-MAW" behavior.

arrows 3,4 - Upper branch MAWs are always unstable due to the positive eigenvalue associated with the saddle-node bifurcation. The resulting incoherent dynamics has been studied quite extensively in the context of hole-defect dynamics [25,31. (i) When a perturbation has pushed an upper branch MAW towards the "lower" part of the bifurcation diagram, the structure decays towards lower branch MAWs (arrow 3). An example of a space time plot for the decay towards a lower branch MAW is shown in Fig. 10a. (ii) When the perturbation pushes the MAW towards the "upper" side of the diagram, the phase gradient peak that characterizes MAWs grows without bound, and at the same time the minimum of $|A|$ approaches zero: a defect is formed (arrow 4). The dynamics after such a defect has formed depends on the values of the coefficients $c_{1}$ and $c_{3}$. Two different examples are shown in Fig. 10 b,c. For more details see section 3 .

arrow 5 - So far we have encountered two scenarios: if the phase gradient peak of a structure is "larger" than that of an upper branch MAW, then it will grow out to form defects. If it is 
"smaller", it will decay back in the direction of the lower branch MAWs. The latter process frequently occurs in phase chaos, preventing the formation of defects, while the former process needs to be initiated by appropriate initial conditions. However, when the upper and lower branches approach each other and disappear in a saddle-node bifurcation, there are no structures left to prevent arbitrary small perturbations to grow out to defects. This dynamical process, which is represented by arrow 5 in Fig. 8 , is the core of our argument: defect formation takes place beyond the saddle-node bifurcation.

\subsection{Breakdown of phase description}

An alternative approach to describe the creation of defects from phase chaotic states is via blow-ups in so-called phase-equations [20]. Phase equations are based on the observation that close to the onset of phase chaos (near the Benjamin-Feir-Newell curve) the amplitude is "slaved" to the phase dynamics. In this situation a phase equation can be obtained by a gradient expansion [4]. The expansion including all parity-symmetric terms up to fourth order [20] reads

$$
\begin{aligned}
\frac{\partial \phi}{\partial t}= & \Omega_{2}^{(1)} \frac{\partial^{2} \phi}{\partial x^{2}}+\Omega_{2}^{(2)}\left(\frac{\partial \phi}{\partial x}\right)^{2}+\Omega_{4}^{(1)} \frac{\partial^{4} \phi}{\partial x^{4}} \\
& +\Omega_{4}^{(2)} \frac{\partial \phi}{\partial x} \frac{\partial^{3} \phi}{\partial x^{3}}+\Omega_{4}^{(3)}\left(\frac{\partial^{2} \phi}{\partial x^{2}}\right)^{2}+\Omega_{4}^{(4)}\left(\frac{\partial \phi}{\partial x}\right)^{2} \frac{\partial^{2} \phi}{\partial x^{2}}
\end{aligned}
$$

where $\Omega_{2}^{(1)}=1-c_{1} c_{3}, \Omega_{2}^{(2)}=-\left(c_{1}+c_{3}\right), \Omega_{4}^{(1)}=-c_{1}^{2}\left(1+c_{3}^{2}\right) / 2, \Omega_{4}^{(2)}=-2 c_{1}\left(1+c_{3}^{2}\right), \Omega_{4}^{(3)}=-c_{1}(1+$ $\left.c_{3}^{2}\right), \Omega_{4}^{(4)}=-2\left(1+c_{3}^{2}\right)$. The lowest order description of phase chaos is obtained when the parameters $\Omega_{4}^{(2)}, \Omega_{4}^{(3)}$ and $\Omega_{4}^{(4)}$ are set equal to zero; the resulting equation is known as the KuramotoSivashinsky equation [2].

The phase equations with higher order terms included have been studied via direct integration by Sakaguchi [20]. For the full Eq. (4), Sakaguchi observed finite time divergences of the phase gradient for coefficients close to the transition from phase to defect chaos in the CGLE. He attributed such divergences to the occurrence of defects in the CGLE. No blow-up of the phase gradient is observed for Eq. (4) without the last term, or for the simple Kuramoto-Sivashinsky equation. Recently, Abel et al. [41] quantified the increasing discrepancies between the phase equations of different orders and the full dynamics in the CGLE with increasing distance from the Benjamin-Feir-Newell curve and identified the relative importance of the various terms in Eq. (四).

Since the essential ingredient of our theory is the occurrence of a saddle-node bifurcation, we have investigated the bifurcation scenario for various truncations of the phase equations (田). In the context of phase dynamics, our Ansatz (2) becomes of the form

$\phi(x, t)=\tilde{\phi}(x-v t)+\left(\omega-c_{3}\right) t$.

We have studied MAW-like structures occurring in the phase equations by employing the same methodology as for the CGLE; the average phase gradient value $\nu$ is fixed to 0 and $P$ parameterizes the spatial period of the MAW. In Fig. 11 we compare bifurcation diagrams and MAW profiles for different expansions at the parameters $c_{1}=3.5, P=50$.

For all phase equations considered here the coherent structures are again born in a Hopf and undergo a drift pitchfork bifurcation, beyond which the maximal phase gradients increase. This leads to increasing discrepancies between different approximations. In particular, the coherent structures for Eq. (包) exhibit saddle-node bifurcations at parameter values not far from those for corresponding MAWs in the CGLE; nevertheless the MAWs of Eq. (四) deviate substantially from the CGLE MAWs for the upper branch of MAWs. The Kuramoto-Sivashinsky equation, 

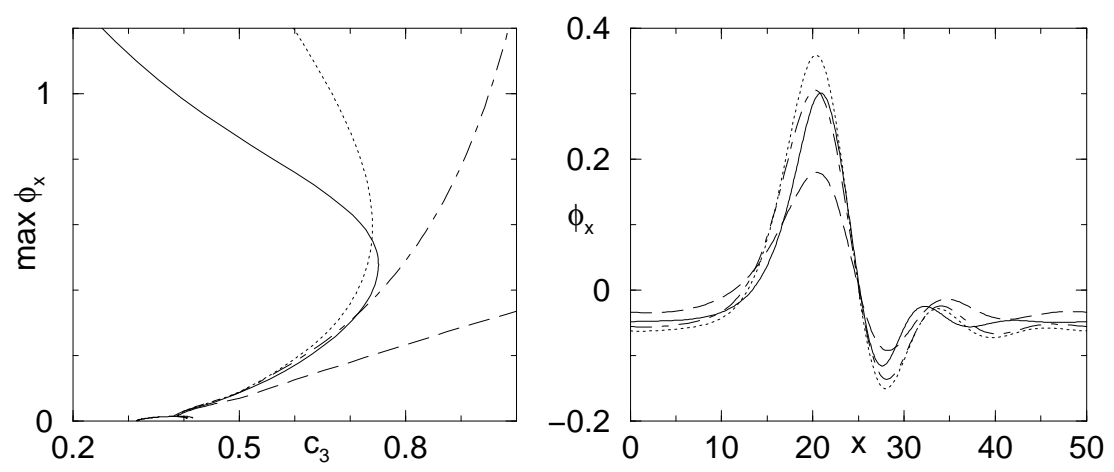

Figure 11. Comparison of different phase expansions : Eq.(岛) (dotted), Eq.(4) without the last term (dot-dashed), Kuramoto-Sivashinsky Eq. (dashed) and CGLE (full curve). Parameters are $c_{1}=3.5, \nu=0, P=50$. (a) Bifurcation diagrams and (b) spatial profiles of lower branch coherent structures at $c_{3}=0.7$.

and Eq. (41) without the last term, do not exhibit a saddle-node bifurcation. Since these latter two models do not experience blow-up, we can safely conclude that these observations confirm our picture, and that the saddle-node bifurcations of coherent structures play the same crucial role in both the full CGLE and its phase equations.

\section{Large scale chaos}

In this section we will study the dynamical evolution of the CGLE near the transitions from phase to defect chaos. The transition between these two states can either be hysteretic or continuous: in the former case, the transition is referred to as $L_{3}$, in the latter as $L_{1}$ [42].

How are defects generated from phase chaos? Let us start to consider a small system in which a stable lower branch MAWs has been created. When we fix the coefficients $c_{1}$ and $c_{3}$ and steadily increase the size of the system, and hence the period $P$ of the MAW, we find that as soon as we push $P$ beyond $P_{S N}$, the MAW structure blows up to form defects. An example of this is shown in Fig. 12a. In a similar fashion, defects are created when the system size $L$ is fixed, and either $c_{1}$ or $c_{3}$ are increased until $P_{S N}<L$ (Fig. 1 $1 \mathrm{c}, \mathrm{d}$ ).

How is this related to phase chaos? As shown in Fig. 12 b, typical phase chaotic states show much more incoherent dynamics, containing many MAW like structures but of much smaller period. Our central conjecture is therefore that the transitions from phase to defect chaos are triggered by the occurrence of near-MAW structures in a phase chaotic state with $\nu=0$ [33] and periods larger than $P_{S N}$, the spatial period of the critical nucleus for defect creation.

To test this conjecture, we have numerically investigated the distribution of inter-peak spacings $p$ of the phase gradient profile (see Fig. 1e,f). In section 3.1 we discuss the definition of $p$ and the details of our numerical analysis. In particular, we have examined in the $c_{1}, c_{3}$ plane 17 different "cuts" across the $L_{1}$ and $L_{3}$ transition lines. In section 3.2 the results of our numerics along a cut through the $L_{1}$ transition line are presented, while section 3.3 is devoted to the $L_{3}$ transition. We will show that the presence of inter-peak spacings $p$ larger than $P_{S N}$ accurately predicts the transition from phase to defect chaos (Fig. 2). In the last section 3.4 we will show that a reasonable, parameter-free estimate of the numerically observed transitions can be obtained via a linear stability analysis of the MAWs. 


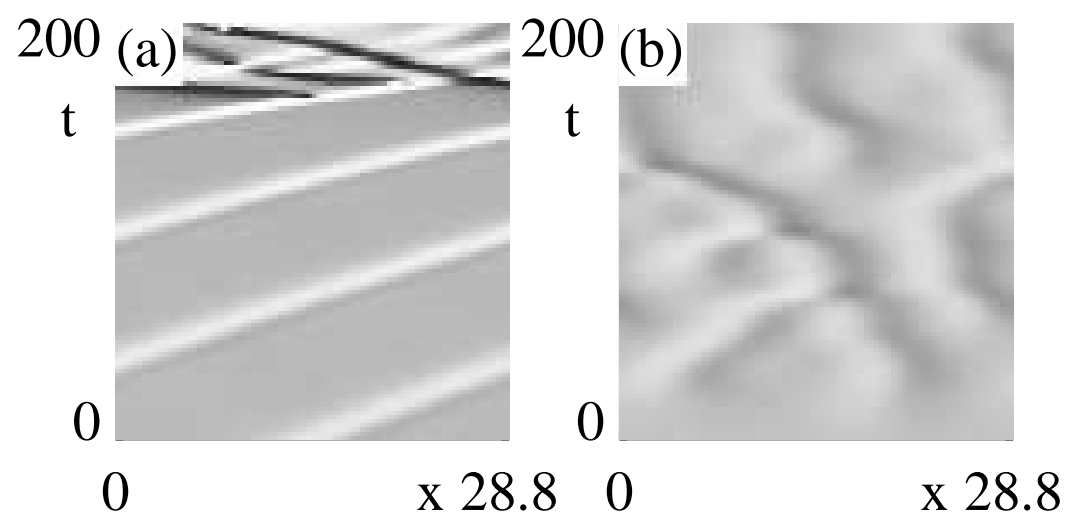

Figure 12. Defect formation at $c_{3}=2, c_{1}=0.7$. (a) Defect formation. As initial condition we took a lower branch MAW with $P<P_{S N}=26.8$ which we embedded in a background of zero wavenumber. The system size $L$ here is equal to 28.8 , which is larger than $P_{S N}$ and a defect is formed; for $L<P_{S N}$ this defect formation does not take place. (b) Random initial conditions in general evolve to MAW like structures with $P<P_{S N}$ which do not lead to defects; the "critical" nucleus that leads to defect formation has a rather small basin of attraction here.

\subsection{Identification of MAWs in the phase-chaotic regime}

To verify our main conjecture, we have to characterize the MAW structures occurring in the phase-chaotic regime. In general this is a complicated task, since the phase gradient profile of a typical phase chaotic state (see Fig. 1le,f and 13) consists of many peaks of different size, spacing and shape; a priori it is unclear how to compare these to MAW profiles. However, a close inspection of the defect forming process reveals that while closely spaced phase gradient peaks evolve in a quite erratic way, well spaced peaks appear to have a more regular dynamics and frequently their overall shape resembles that of MAWs (see Fig. 13). These large period near-MAWs modify their shape quite slowly with respect to the other structures present in the chaotic field, and propagate over a disordered background. Therefore we study the distribution of inter-peak distances $p$, keeping in mind that the tail of this distribution is relevant for defect generation.

The phase gradient profile of a coherent MAW (see Figs. 1 a and Fig. 5a) shows a secondary maximum. To obtain the correct period $P$ of a near-MAW, such small extrema should be neglected when the inter-peak spacing $p$ is measured. We introduce a cutoff for the size of the phase gradient peak equal to the size of the secondary extremum of the MAW with the largest $P$. As an additional result of this cutoff, small fluctuations are not considered as MAW peaks. It should be noted that the tail of the distribution of $p$ is rather insensitive to the precise value of this cutoff.

In order to estimate the probability density $D(p)$, for every time interval $\tau=0.5$, the interpeak periods $p$ of the spatial profile of the phase gradient are determined. In addition, for every snapshot the largest value $p_{\max }$ of the inter-peak spacing $p$ is stored separately, and this leads to the distribution $D\left(p_{\max }\right)$. From the spatial profile of $|A|$ the distribution $D(|A|)$ and the minimal amplitude value $|A|_{\text {min }}$ can be derived. This latter quantity is used to detect defects: when $|A|_{\min }$ falls below a value of 0.1 , we take this as an indication of a defect.

Extensive simulations have been made possible thanks to an innovative time-splitting code which ensures precision and stability comparable with pseudo-spectral codes, but is noticeably faster [23]. The spatial resolution $\Delta x$ has been set to 0.5 and the integration time step to 


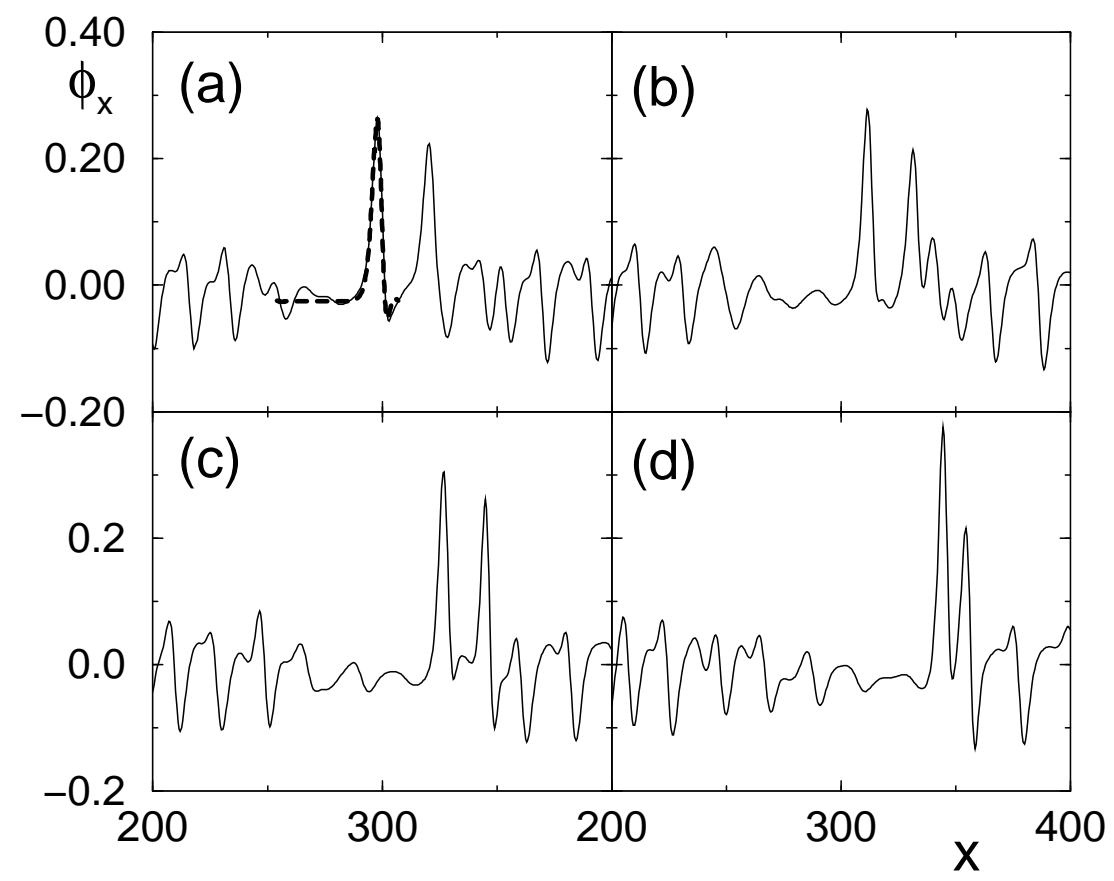

Figure 13. Local phase gradient of the chaotic field just before defect formation for $c_{1}=0.65$ and $c_{3}=2$. Panel (a) is a snapshot of the field at a time $t=120$ before the occurrence of the defect, (b), (c) and (d) are successive snapshots taken at time intervals $\delta t=30$. In (a) also the shape of the MAW at the saddle-node is superimposed (thick dashed line) on the profile.

0.05. Simulations have been carried out for integration times ranging from $t=5 \times 10^{5}$ to $t=3 \times 10^{7}$ and for a typical system size $L=512$; occasionally, runs have been performed with $L=100,200$ and 5000. Typically, our runs start from random initial conditions of the type $A_{k}(t=0)=|A|_{k}(t=0) \cdot \mathrm{e}^{i \phi_{k}(t=0)}\left(\right.$ where $A_{k}(t)=A(k \Delta x, t)$ and $\phi_{k}(t)=\phi(k \Delta x, t)$ ) with

$$
|A|_{k}(0)=1+r_{k}
$$

$\phi_{k}(0)=\phi_{k-1}(0) * 0.8+q_{k}$

where $r_{k}$ and $q_{k}$ are random numbers uniformly distributed in $[-0.05,+0.05]$ and $\phi_{1}(0)=0.0005$. This initial condition (7) leads to a smooth phase and the formation of defects due to initial discontinuities is avoided.

In sections 3.2 and 3.3 we will consider in detail two particular cuts in the $\left(c_{1}, c_{3}\right)$ coefficient space, one across the $L_{1}$ and one across the $L_{3}$ curve. In particular, we will analyze the behavior of the probability densities $D(|A|), D(p)$ and $D\left(p_{\max }\right)$ for both transitions.

\section{2. $L_{1}$ transition}

In this section we concentrate on the $L_{1}$ transition that is observed when the value of $c_{1}$ is fixed at 3.0 and $c_{3}$ is varied.

Transition to defect chaos - Starting from random initial conditions we have integrated the dynamics of the CGLE for long durations. For a fixed system size $L$ we observe that, as a function of the total integration time, the value of $c_{3}^{*}$ for which defects are formed appears to decrease. Similar behavior occurs when the system size $L$ is increased for fixed integration times. For example, for an integration time of $3 \times 10^{7}$ and $c_{1}=3$ we find for system size 100, 200 and 512 critical values $0.82,0.81$ and 0.79 , respectively. For a size $L=5000$ and integration times $3 \times 10^{6}$ a critical value of 0.79 is also found. 
Note that even the lowest value of $c_{3}^{*}$ for the numerically measured transition obtained here is far above the lower bound $c_{3}^{\infty}=0.704$ which is the value of $c_{3}$ where the size of the critical nucleus for defect formation diverges $\left(P_{S N} \rightarrow \infty\right)$. Below, we will give an estimate of the critical value $\hat{c}_{3}$ for which the defect density should vanish in the thermodynamic limit by extrapolating finite time and finite size data.

Distribution of $p$ - Let us now consider the distribution of $p$ 's for various coefficients $c_{3}$ near the $L_{1}$ transition. It is clear from the data reported in Fig. 14 that the shape of these distributions is quite insensitive to the presence or absence of defects. This can be partly explained by the fact that just above the $L_{1}$ transition defects arise in the system as rare isolated events occurring during the spatio-temporal evolution, as shown in Fig. 10c. This is fully consistent with earlier observations that the $L_{1}$ transition is continuous 17,19,21]. We focus on the tail of the probability density $D(p)$, since this gives information on the probability to observe defects. Our numerical results suggest an exponential decay, i.e., $D(p) \propto \exp (-\alpha \cdot p)$ with $\alpha=0.6$ for sufficiently large $p$.

Similarly to the apparent transition value $c_{3}^{*}$, the values associated to extremal events $|A|_{\min }$ and $p_{\max }$ depend on integration times and system sizes. By assuming that $D(p)$ remains finite (but likely exponentially small) for large $p$, we can expect that for long enough times, rare events associated with large values $p$ will occur, and hence, defects can form after possibly very long transients.

Crossover behavior - A good order parameter to identify the occurrence of the transition starting from the defect chaos phase near the $L_{1}$ transition is the defect density $\delta_{D}$ which measures the number of defects occurring per space and time unity. In the defect chaos regime $\delta_{D}>0$, while it vanishes at the $L_{1}$-transition. Now we can relate this order parameter to the tail of the distribution of $p$. Our conjecture states that defects should arise when $p>P_{S N}$, therefore the defect density $\delta_{D}$ should be related to the probability to have structures of period $p>P_{S N}$, i.e.,

$\delta_{d} \propto \int_{P_{S N}}^{\infty} d p D(p) \propto \mathrm{e}^{-\alpha P_{S N}}$

where $D(p) \propto \exp (-\alpha \cdot p)$ has been used. If we now assume that the distribution $D(p)$ does not vary significantly across the transition (as is evident from Fig. 14), then the change in the probability to have $p>P_{S N}$ is dominated by the changes in $P_{S N}$ with $c_{3}$. A reasonable fit of our bifurcation data for $P_{S N}$ (see Fig. 母) in the interval $30 \leq P_{S N}<300$ is

$P_{S N} \approx \frac{\beta}{c_{3}-c_{3}^{\infty}}$

where $\beta \approx 4.38$. Combining this result with the Ansatz (8), we immediately obtain the following expression for the defect density:

$\delta_{d} \propto \mathrm{e}^{-\alpha \beta /\left(c_{3}-c_{3}^{\infty}\right)}$.

A similar expression was proposed in [17,21] for the defect density near the $L_{1}$ transition.

In order to verify if the expression (10) is reasonable also for our choice of the parameters, we have estimated the probability [23]

$w(|\hat{A}|)=\int_{0}^{|\hat{A}|} d|A| D(|A|)$,

to observe an amplitude less than $|\hat{A}|$. This quantity gives a more precise characterization of the $L_{1}$-transition than $\delta_{D}$, because it measures not only the extreme events corresponding to true 

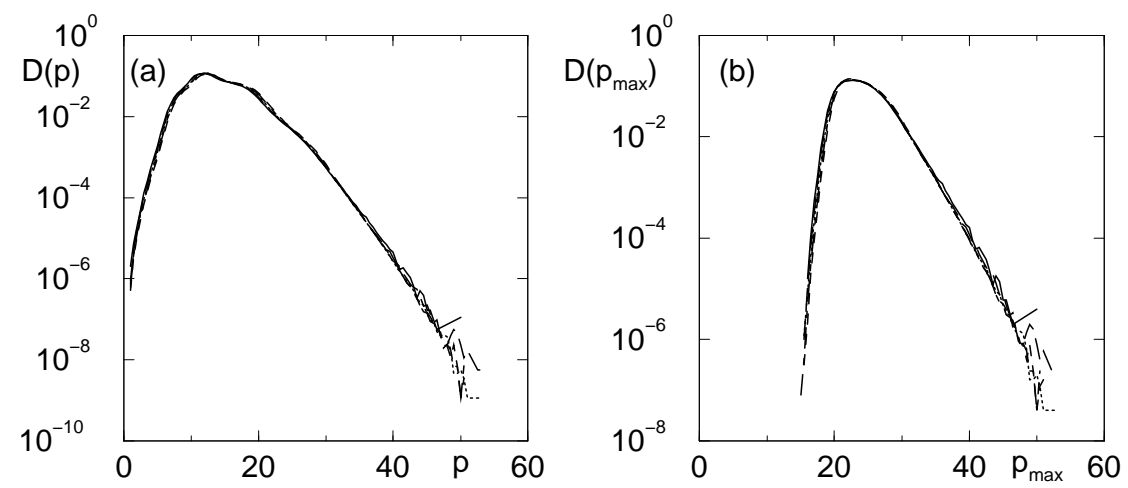

Figure 14. Probability densities (a) $D(p)$ and (b) $D\left(p_{\max }\right)$ for $c_{1}=3$ and various values of $c_{3}$ on a lin-log scale. The curves refer to $c_{3}$ below $c_{3}^{*}=0.79$ (namely to $c_{3}=0.77$ and 0.78 ), as well as to values corresponding to the defect chaotic regime: $c_{3}=0.79,0.80$ and 0.81 . The system size was $L=512$ and the integration times where $t=5 \times 10^{5}$ for $c_{3}=0.81, t=5 \times 10^{6}$ for $c_{3}=0.80$ and $t=25 \times 10^{6}$ for all other values.
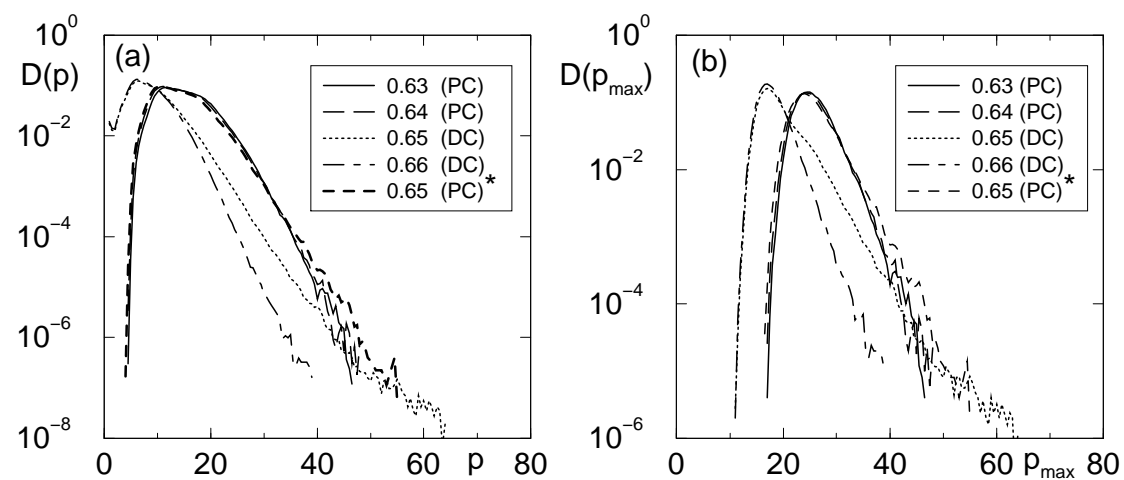

Figure 15. Probability densities (a) $D(p)$ and (b) $D\left(p_{\max }\right)$ for $c_{3}=2$ and various values of $c_{1}$ reported in a lin-log scale. The data are for a system size $L=512$ and for integration times ranging from $t=5 \times 10^{5}$ for $c_{1}=0.63,0.64,0.66$ and $0.65(\mathrm{PC})^{\star}$ to $t=2.5 \times 10^{6}$ for $c_{1}=0.65$ (DC). The labels DC and PC indicate that we are in presence or absence of defects, respectively. The label $(\mathrm{PC})^{\star}$ refers to the regime before defect formation at $c_{1}=0.65$.

defects, but also the tendency of the system to generate structures characterized by small $|A|_{\text {min }}$. We estimated the quantity (11) for several $|\hat{A}|$ values and for various $c_{3}$ parameter values in the defect chaos regime. Reporting $\ln [w(|\hat{A}|)]$ as a function of $1 /\left(c_{3}-\hat{c}_{3}\right)$ a reasonable linear scaling is observed in the range $0.795 \leq c_{3} \leq 0.85$, for $0.1 \leq|\hat{A}| \leq 0.5$, with the choice $\hat{c}_{3}=0.72$. The value $\hat{c}_{3}$ where the defect density should asymptotically vanish is much smaller than $c_{3}^{*}$ obtained via direct numerical simulations but still bigger than $c_{3}^{\infty}=0.704$ where $P_{S N} \rightarrow \infty$.

We can now easily estimate the integration time needed to observe a tiny shift of the apparent value $c_{3}^{*}$ towards the corresponding asymptotic value $c_{3}^{\infty} \approx 0.704$. Limiting our analysis to system size $L=512$, a typical time-scale to observe a defect at $c_{3}=0.79$ is $t \sim 3 \times 10^{7}$. At this value of $c_{3}, P_{S N}=46.5$, while for $c_{3}=0.739, P_{S N}=105$. Invoking the exponential decay of $D(p)$, one immediately finds that the time scale to observe a defect at $c_{3}=0.739$ is of order $10^{17}$, which is completely outside the reach of present day computers. 

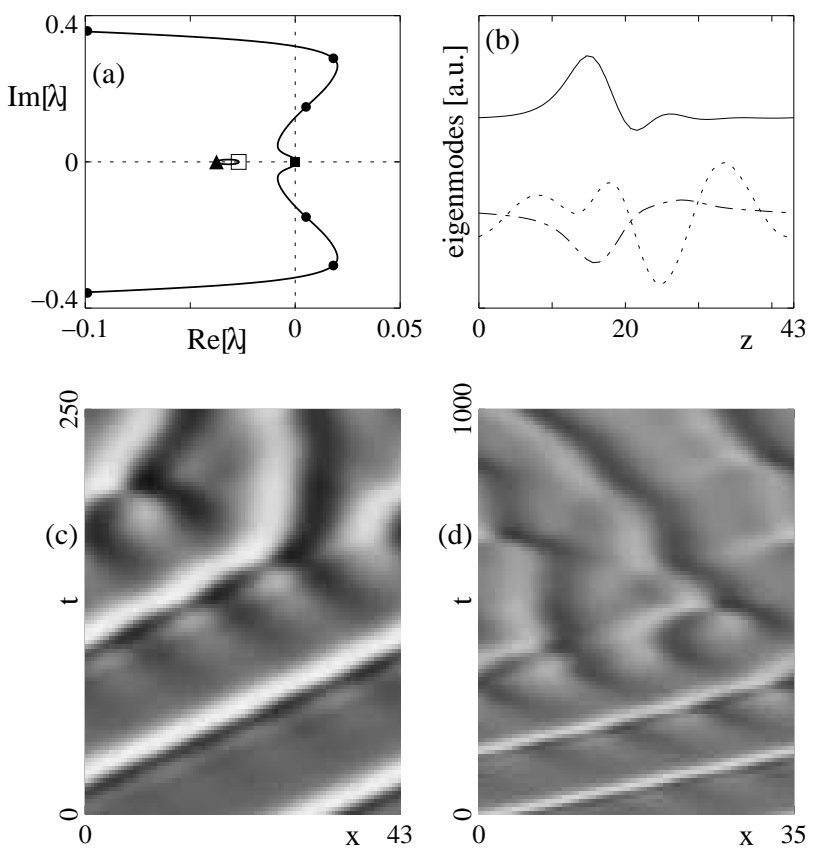

Figure 16. Results of the linear stability analysis : (a) leading part of the eigenvalue spectrum (continuous spectrum denoted by the full curve, Goldstone modes by a filled square, saddlenode by filled triangle, interaction by open square and splitting modes in $L=P$ by dots, respectively), (b) splitting eigenmodes (dot-dashed and dotted, $\lambda_{\text {split }}=0.018 \pm 0.28 i$ ) of the phase in $L=P$ compared with spatial MAW profile of the phase gradient (full curve). (c,d) Space time plots showing the splitting of a MAW initially perturbed by small noise. Parameters are $c_{1}=3, c_{3}=0.72, P=43$ near $L_{1}$ for $(\mathrm{a}-\mathrm{c})$ and $c_{1}=0.65, c_{3}=2, P=35$ near $L_{3}$ for $(\mathrm{d})$.

\section{3. $L_{3}$ transition}

In order to characterize the $L_{3}$ transition from phase to defect chaos in more detail $c_{3}=2$ has been fixed, while the coefficient $c_{1}$ is varied. The $L_{3}$ transition is hysteretic [17, 19]: to the left of $L_{3}$ one may have phase or defect chaos depending on the initial conditions. Beyond the $L_{3}$ phase chaos breaks down and defects occur spontaneously for any initial condition. In order to study the dynamics across this transition we therefore initialized the simulations with initial conditions (6),(7) or used relaxed phase chaos configurations corresponding to values of $c_{1}$ far below the $L_{3}$ line.

The probability densities $D(p)$ and $D\left(p_{\max }\right)$ are shown in Fig. 15. For $c_{1}<c_{1}^{*}=0.65$ all distributions collapse on a unique curve, but as soon as defects arise the distributions change substantially. Whenever a defect is generated, hole-defect dynamics takes place (see Fig. 10b). As a result phase chaos is replaced by defect chaos. The noticeable modification of the distributions thus reflects the fact that the $L_{3}$ transition is discontinuous. Also the probability density for $|A|$ changes abruptly across the $L_{3}$ transition.

\subsection{Mechanism for the selection of $p$}

When approaching the transition to defect chaos from the Benjamin-Feir-Newell curve, three parameter regions, corresponding to different dynamical regimes, can be distinguished (Fig. 18). The first encountered region corresponds to infinite values of $P_{S N}$ : here we expect no defects to occur, irrespectively of system size and integration time. The phase chaos is the asymptotic 

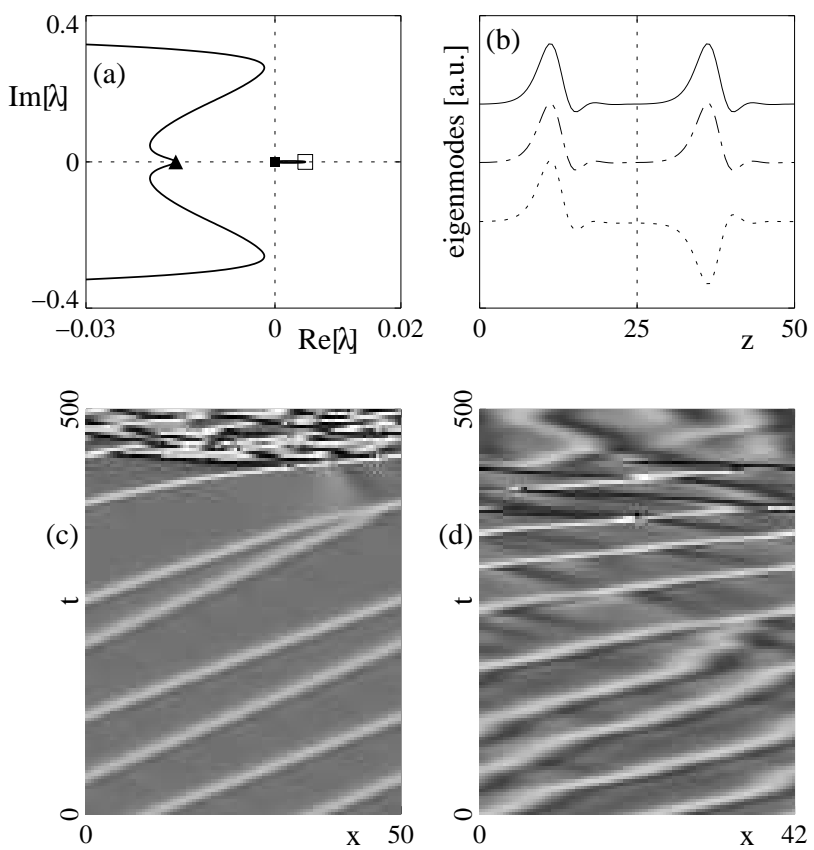

Figure 17. Results of the linear stability analysis : (a) leading part of the eigenvalue spectrum (continuous spectrum denoted by the full curve, Goldstone modes by a filled square, saddle-node by filled triangle and interaction by open square at $\lambda_{i n t}=+0.0048$, respectively), (b) Goldstone mode (dot-dashed) and interaction eigenmode (dotted curve) for the phase compared with spatial MAW profile of the phase gradient (full curve) in $L=2 P$. (c,d) Space time plots showing the attraction of two periods of the same MAW initially perturbed by the interaction eigenmode. Parameters are $c_{1}=0.7, c_{3}=2, P=25$ near $L_{3}$ for $(\mathrm{a}-\mathrm{c})$ and $c_{1}=3, c_{3}=0.85, P=21$ near $L_{1}$ for $(d)$.

regime in this first region. Then, when $c_{1}$ and/or $c_{3}$ are increased, a crossover regime is reached where extreme events (large inter-peak spacings) may lead to defect formation. Here phase chaos can persist as a long lived transient, but eventually we expect it to break down. Then, when $c_{1}$ and/or $c_{3}$ are even further increased, we experience a dramatic drop in transient times, and defect chaos sets in quite rapidly. We understand this drop to occur when typical values of $p$ (and not rare extreme events) become larger than the corresponding $P_{S N}$ values.

An approximate prediction for the location of the apparent phase to defect chaos transition (numerically obtained from the defect density) can be achieved in terms of a simple linear stability analysis of the MAWs (Figs. 16 and 17). A key element in our framework is the "typical large value" of $p$ as a function of coefficients $c_{1}$ and $c_{3}$; below we will identify two linear instabilities that act to either increase or decrease $p$, and their balance sets a scale for typical $p$ that will predict the location of the transition from phase to defect chaos rather well.

Due to translational and phase symmetries both MAW branches have neutral modes, i.e., Goldstone modes. The eigenvalue associated with the saddle-node bifurcation is positive for MAWs of the upper branch and negative for the lower branch. In what follows the lower branch MAWs are considered exclusively.

Splitting - The spatial structure of a MAW of large period consists, roughly, of a homogeneous plane wave part and a local peak part. For the parameter regime we consider here, fully extended plane waves are linearly unstable, and so we may expect that the MAW spectrum will be 
dominated by this instability for sufficiently large values of $P$. Our linear stability analysis indeed shows that for appropriate parameters $(L=P)$ and small enough $P$, all eigenvalues $\lambda_{i}<0$, but when we increase $P$, MAWs become linearly unstable $\left(\lambda_{\text {split }}>0\right.$, Fig. 16). The shape of the unstable eigenmodes (Fig. 16 b) suggests that this instability leads to the growth of a new peak in the homogeneous part of the MAW, and this is indeed the behavior observed in numerical simulations of the perturbed MAW (Fig. 16 c,d). As a result two (or more) short MAWs with smaller $P$ will appear. We interpret this process as the splitting of a MAW in two or more smaller MAWs and we call the eigenmodes associated to such instability "splitting modes".

Clearly, this instability tends to reduce the peak-to-peak distances $p$ and prevents MAWs to cross the SN boundary; in the phase chaotic regime this instability tends to inhibit defect generation.

Interaction - By using a Bloch Ansatz [43], we extended the stability analysis to systems with $n$ identical pulses $(L=n P)$. For $n>1$, an additional instability may appear 444 (see Fig. 17). Eigenvalues $\lambda_{\text {int }}>0$ are found mainly for small $P$ (typically $P<30$ ). The shape of the eigenmodes, i.e., an alternating sequence of positive and negative translational Goldstone modes (Fig. 17b), suggests that the instability is due to the interaction between adjacent MAWs. This interaction shifts adjacent peaks into opposite directions, thereby creating occasional larger values of $p$ (Fig. 177c,d). In phase chaos this process leads to an increase of the spacing $p$ between some peaks, thus enhancing the generation of defects.

Competition of Instabilities - Both the splitting and interaction mechanisms are similar to instabilities observed in the Kuramoto-Sivashinsky equation [4.40]. We believe that phase chaos is governed by the competition of these two mechanisms that tend to increase or decrease the inter-peak spacings $p$. Almost independent of the coefficients the splitting instability dominates for MAWs with $P>30$. This can explain why large inter-peak spacings $p>30$ become rare as reported in Figs. 14, 15.

We suggest a connection between the interchanging dominance of these two different instabilities and the sudden change of $\delta_{D}$ (near $L_{1}$ ) or the transient times before defect occurrence (near $L_{3}$ ). We calculated the linear stability spectra for a variety of coefficients and periods $P$ close to $P_{S N}$. From these we obtain a curve in coefficient space (Fig. 18) where the real parts of interaction and splitting eigenvalues are equal. For larger $c_{1}$ or $c_{3}$, interaction becomes stronger, and we expect larger $p$ 's and defect formation, while for smaller $c_{1}$ and $c_{3}$, splitting dominates, $p$ 's are decreased and defect formation becomes rare.

As shown in Fig. 18, the curve where the two instabilities are equally strong near the saddlenode bifurcation gives a rather good estimate of where the apparent transition from phase to defect chaos occurs. Notice that in this "balance of instabilities" picture, there is no tunable parameter: once we have calculated $P_{S N}$ and the instabilities of the MAWs for a range of coefficients, a precise prediction for the "transition" from phase to defect chaos can be given.

\section{Discussion and Final Remarks}

In this section we report some open questions related to defect formation, together with some final remarks and a brief outlook.

Further Refinements - In order to accurately test our results, we have measured for each of the 17 cuts and for several values of the coefficients across the $L_{1}$ - or $L_{3}$-lines the amplitude distribution $D(|A|)$ and the phase gradient peak-to-peak spacing distribution $D(p)$. We conjectured that defects occur if and only if $p>P_{S N}$. Indeed, we observe that in 11 out of 17 points such conjecture is fulfilled. On the remaining 6 points the theoretical conjecture leads to 


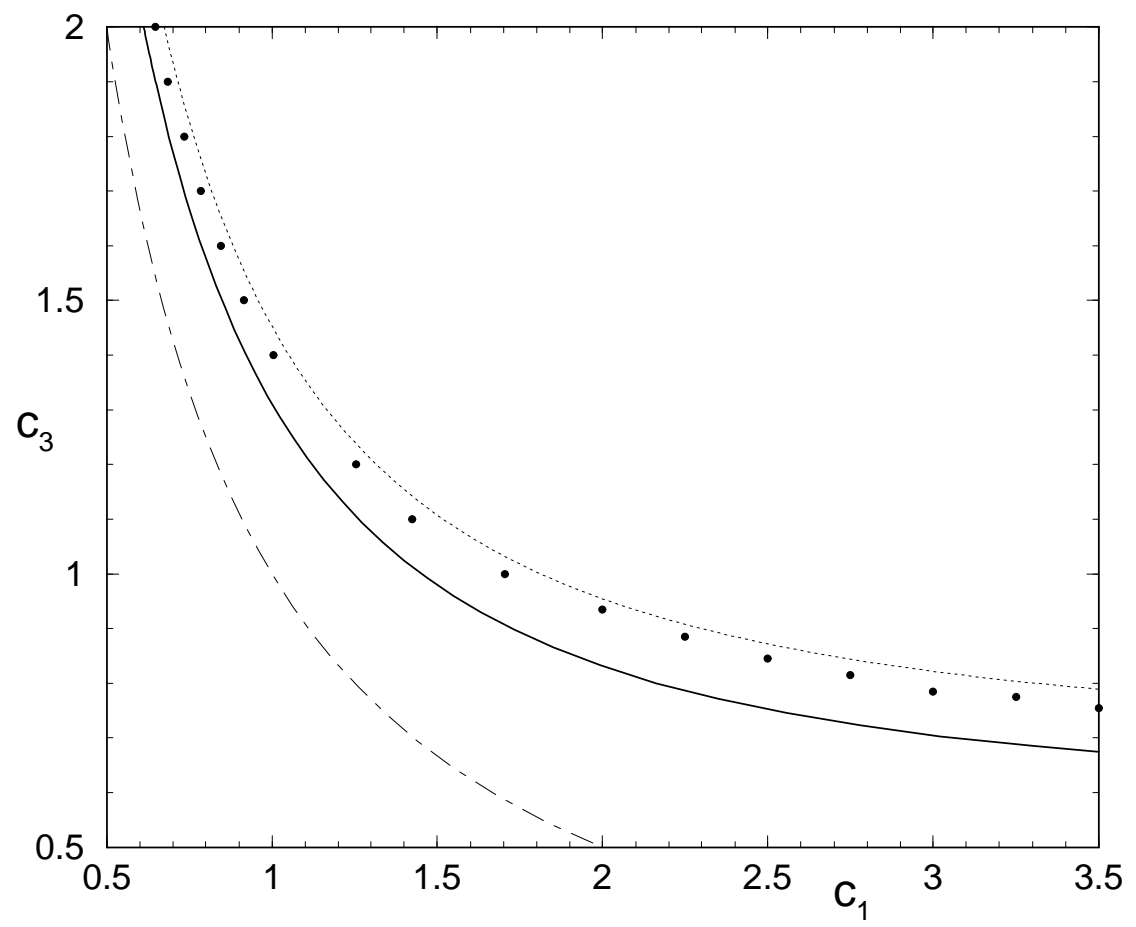

Figure 18. Space of $\left(c_{1}, c_{3}\right)$-coefficients for the CGLE with the Benjamin-Feir-Newell curve (dot-dashed), the lower bound $P_{S N} \rightarrow \infty$ (full) and the stability-based estimate (dotted) for the transition from phase to defect chaos. The symbols refer to our numerical data for the appearance of defects.

an estimation of the transition lines within a maximal error bar of $3 \%$. The points determined following the conjecture are indicated as empty circles in Fig. 2. The small deviations may have different reasons, that we summarize below:

(i) If fluctuations occurring during the phase chaotic dynamics are only moderate, such as happens near the $L_{3}$ transition line or for small system sizes, more complex coherent structures can survive for a short time. Here we analyzed only the shortest coherent structures characterized by a single hump. We believe that this is sufficient to understand the main aspects of the dynamics of large systems. However, longer combined MAWs with more than one hump emerge from periodic MAWs via period doubling bifurcations. The existence of the long combined MAWs is limited by saddle-node bifurcations analogously to single MAWs, but these bifurcations occur at slightly bigger values of the parameters $c_{1}$ and $c_{3}$. Therefore the appearance of these more complicated structures can delay defect formation even if one inter-peak spacing within the structure is bigger than $P_{S N}$ of the single MAW.

(ii) Near the $L_{1}$ line the dynamical fluctuations in the phase chaotic regime are stronger than in the proximity of the $L_{3}$ line. In this case and for sufficiently high values of the parameter $c_{1}$ we observed situations where not only the structure with the longest inter-peak spacing but also the neighboring structures were involved in the defect formation.

(iii) The assumption to consider MAWs with $\nu=0$ is only an approximation. If the average phase gradient locally (on scales $P$ ) deviates from 0 then the saddle-node bifurcation slightly shifts towards smaller coefficients [34].

As far as the (numerically) improved $L_{3}$ and $L_{1}$ lines are concerned, we observe that both these lines lie to the left of the ones determined in earlier numerical studies [19. This is due 
to the fact that our simulations are of longer duration then those performed previously. This confirms the expectation that such transition lines will shift towards the Benjamin-Feir-Newell curve for increasing systemsize and integration times [21]. Moreover, some authors claim that indeed in the thermodynamic limit $L_{1}$ and $L_{3}$ will coincide with the Benjamin-Feir-Newell curve and the phase chaos regime will disappear [27]. On the basis of our simulations we cannot exclude such a possibility for higher space dimensions, but based on the results presented in this paper we conjecture that the saddle-node line for $P \rightarrow \infty$ provides a lower boundary for the transition from phase to defect chaos in the one-dimensional CGLE.

Final Remarks - We have presented a systematic study of modulated amplitude waves (MAWs) in the complex Ginzburg-Landau equation (CGLE). These periodic coherent structures originate from supercritical bifurcations from the homogeneous oscillation of the CGLE due to the Benjamin-Feir instability. The range of existence of MAWs is bounded by saddle-node bifurcations occurring for values of $c_{1}$ and $c_{3}$ that depend on the period $P$ of the MAWs. Approaching the transition from phase to defect chaos, near-MAWs with large $P$ occur in phase chaos, and defects are generated when the period of these near-MAWs becomes larger than the spatial period $P_{S N}$ of the critical nucleus. This scenario is valid for both the $L_{1}$ and $L_{3}$ transition. The divergence of $P_{S N}$ for coefficients in the phase-chaos regime led us to conjecture that there is a lower bound for the transition from phase to defect chaos. Considerations of the linear stability properties of MAWs in light of their tendency to increase or decrease the typical period $p$ in phase chaos, has led us to a fit-free estimate of the apparent transition from phase to defect chaos that fits the numerical data well.

Altogether, our study leaves little space for doubt that the transition from phase chaos to defect chaos in the CGLE is governed by coherent structures and their bifurcations. From a general viewpoint, our analysis shows that there is no collective behavior that drives the transition. Instead, strictly local fluctuations drive local structures beyond their saddle-node bifurcation and create defects.

Outlook - We want to stress here that the extension of the analysis to MAWs with nonzero average phase gradients [34], will be of considerable interest for experimentalists, because in some recent experiments concerning Rayleigh-Bénard or Marangoni convection in quasi-onedimensional geometries, supercritical Eckhaus instabilities of plane wave trains and the corresponding emergence of stable saturated MAWs have been observed [13] 15]. These states are analogous to what happens for the 1d CGLE when phase chaotic solutions with $\nu \neq 0$ are considered [22,23].

The relevance of MAWs for two-dimensional structures is suggested by recent experimental evidence of MAWs observed in connection with superspiral and spiral breakup occurring in a Belousov-Zhabotinsky reaction [11]. Moreover, in the phase chaotic regime of the $2 \mathrm{~d}$ CGLE the correspondence between long inter-peak spacings (here diameter of cells) and the strength of the local modulation has already been noticed numerically [27. Additional mechanisms present in $2 \mathrm{~d}$ remain to be explored. Thereby it might turn out that phase chaos exists in the thermodynamic limit in $1 \mathrm{~d}$ only but not in $2 \mathrm{~d}$ as previously conjectured [27].

It is a pleasure to acknowledge discussions with H. Chaté, M. Howard and L. Kramer. AT and $\mathrm{MB}$ are grateful to ISI Torino for providing a pleasant working environment during the Workshop on "Complexity and Chaos" in October 1999. AT would also thank Caterina, Daniel, Katharina and Sara for providing him with a faithful representation of a chaotic evolution. MGZ is supported from a post-doctoral grant of the MEC (Spain) and FOMEC-UBA (Argentina). $\mathrm{MvH}$ acknowledges financial support from the EU under contract ERBFMBICT 972554. 


\section{REFERENCES}

1. L. Brusch, M. G. Zimmermann, M. van Hecke, M. Bär and A. Torcini, Phys. Rev. Lett. 85, 86 (2000).

2. Y. Kuramoto, Chemical Oscillations, Waves and Turbulence (Springer, 1984, Berlin).

3. M. C. Cross and P. C. Hohenberg, Rev. Mod. Phys. 65, 851 (1993).

4. P. Manneville, Dissipative structures and Weak Turbulence (Academic Press, 1990, San Diego); T. Bohr, M. H. Jensen, G. Paladin and A. Vulpiani, Dynamical systems approach to turbulence (Cambridge Univ. Press) (1998).

5. A recent and detailed review on the CGLE is the following : I.S. Aranson and L. Kramer, The World of Complex Ginzburg-Landau Equation, to appear in Rev. Mod. Phys.

6. J. M. Vince and M. Dubois, Physica D, 102, 93 (1997).

7. M. Rabaud, S. Michalland and Y. Couder, Phys. Rev. Lett. 64, 184 (1990); D. P. Vallette, G. Jacobs and J. P. Gollub, Phys. Rev. E 55, 4274 (1997).

8. S. Akamatsu and G. Faivre, Phys. Rev. E 58, 3302 (1998).

9. M. Lücke, W. Barten and M. Kamps, Rhysica D, 61, 183 (1992).

10. Y. Liu and R. E. Ecke, Phys. Rev. Lett. 78, 4391 (1997).

11. Q. Ouyang and J. M. Flesselles, Nature 379, 143 (1996); Q. Ouyang, H. L. Swinney and G. Li, Phys. Rev. Lett. 84, 1047 (2000); L. Q. Zhou and Q. Ouyang, Phys. Rev. Lett. 85, 1650 (2000).

12. P. Bot and I. Mutabazi, Eur. Phys. J. B 13, 141 (2000).

13. N. Mukolobwiez, A. Chiffaudel and F. Daviaud, Phys. Rev. Lett. 80, 4661 (1998); J. Burguete, H. Chaté, F. Daviaud and N. Mukolobwiez, Phys. Rev. Lett. 82, 3252 (1999).

14. A. Wierschem, H. Linde and M. G. Velarde, Phys. Rev. E 62, 6522 (2000).

15. B. Janiaud, A. Pumir, D. Bensimon, V. Croquette, H. Richter and L. Kramer, Physica D, 55, 269 (1992).

16. A. Pumir, B. I. Shraiman, W. van Saarloos, P. C. Hohenberg, H. Chaté and M. Holen, p. 173 in C. D. Andereck and F. Hayot (Eds.), "Ordered and Turbulent patterns in Taylor-Couette Flow" (Plenum Press, New York, 1992)

17. B. I. Shraiman, A. Pumir, W. van Saarloos, P. C. Hohenberg, H. Chaté and M. Holen, Physica D 57, 241 (1992).

18. M. V. Bazhenov, M. I. Rabinovich and A. L. Fabrikant, Phys. Lett. A 163, 87 (1994).

19. H. Chaté, Nonlinearity 7, 185 (1994); p. 33 in P. E. Cladis and Palffy-Muhoray (Eds.), "Spatio-Temporal Pattern Formation in Nonequilibrium Complex Systems" (Addison Wesley, Reading, 1995).

20. H. Sakaguchi, Prog. Theor. Phys. 84, 792 (1990).

21. D. A. Egolf and H. S. Greenside, Phys. Rev. Lett. 741751 (1995)

22. R. Montagne, E. Hernández-García and M. San Miguel, Phys. Rev. Lett. 77, 1047 (1996); R. Montagne, E. Hernández-García, A. Amengual and M. San Miguel, Phys. Rev. E 55, 151 (1997).

23. A. Torcini, Phys. Rev. Lett. 77, 1047 (1996); A. Torcini, H. Frauenkron and P. Grassberger, Phys. Rev E 55, 5073 (1997).

24. W. van Saarloos and P. C. Hohenberg, Physica D 56, 303 (1992); 69, 209 (1993) [Errata].

25. M. van Hecke, Phys. Rev. Lett. 80, 1896 (1998).

26. G. Giacomelli, R. Hegger, A. Politi and M. Vassalli, Phys. Rev. Lett. 85, 3616 (2000).

27. P. Manneville and H. Chaté, Physica D 96, 30 (1996).

28. By substituting $\kappa:=a_{z} / a$ one reproduces the form of the ODEs used in [24] which is more appropriate for studies of fronts. 
29. K. Nozaki and N. Bekki, J. Phys. Soc. Jpn. 53 (1984) 1581.

30. For completeness we point out that the ODEs (3) also contain complicated multi-loop orbits that correspond to more complex coherent structures which have a small basin of attraction and little relevance for the dynamics of the CGLE.

31. M. van Hecke and M. Howard, Phys. Rev. Lett. 86, 2018 (2001).

32. G. Hager, Quasiperiodische Lösungen der eindimensionalen komplexen Ginzburg-Landau Gleichung, Diploma Thesis, University of Bayreuth, Germany (1996).

33. The maximal "conserved" (during time evolution) average phase gradient vanishes approaching the transition to defect chaos 22,23]. This result is rigorous only on scales of the system size but for smaller portions $\nu$ can fluctuate around 0 . Typically we observe quasi-coherent structures (near-MAWs) in the phase chaotic regime with associate $\nu$-values in the interval $[-0.01,+0.01]$. MAWs with such small $\nu$ do not deviate much from the $\nu=0$ MAWs [34, therefore the comparison of the observed structures with the $\nu=0$ MAWs is satisfactory.

34. L. Brusch, A. Torcini and M. Bär, in preparation.

35. E. J. Doedel, X. J. Wang and T. F. Fairgrieve, AUTO94: Software for continuation and bifurcation problems in ordinary differential equations, Applied Mathematics Report, California Institute of Technology (1994).

36. The linear stability analysis of the uniformly oscillating solutions $A_{0}(x, t)=\mathrm{e}^{i c_{3} t}$, can be performed by considering the following perturbed solution $A(x, t)=(1+a(x, t)) \mathrm{e}^{i c_{3} t}$ 沺. Where $a(x, t)=\sum_{k} a_{k} \mathrm{e}^{i k x+\lambda_{k} t}$ with $\lambda_{k}=\sigma_{k}+i \Omega_{k}$. It is straightforward to show that the real part of the growth-rate $\sigma_{k}$ is, up to fourth order in $k$, given by

$\sigma_{k} \sim-\left(1-c_{1} c_{3}\right) k^{2}-\frac{1}{2}\left(1+c_{3}^{2}\right) c_{1}^{2} k^{4}$

From this expression it is clear that $\sigma_{k}>0$ only if $c_{1} c_{3}>1$ (this is nothing else than the Newell criterion). Moreover there exists a critical value $k_{0}^{2}=\left[2\left(c_{1} c_{3}-1\right)\right] /\left[c_{1}^{2}\left(1+c_{3}^{2}\right)\right]$ above which $\sigma_{k}$ is always negative. For finite size systems the smallest allowed wavevector is $k_{\min }=2 \pi / L$, therefore the uniform oscillation becomes unstable for $k_{\min } \leq k_{0}$ and this condition allows to derive the corresponding critical values of the parameters $c_{1}$ and $c_{3}$.

37. M. Kness, L. Tuckerman and D. Barkley, Phys. Rev. A 46, 5054 (1992).

38. See chapter 3 of G. Iooss and D. D. Joseph, Elementary Stability and Bifurcation Theory (Springer, Berlin, 1980).

39. An exception on this rule occurs for large $c_{1}$, where the dependence of $c_{3}$ on $P$ at the saddle-node becomes non-monotonic.

40. H.-C. Chang, E. A. Demekhin and E. Kalaidin, SIAM J. Appl. Math. 58, 1246 (1998); H.-C. Chang, E. A. Demekhin and D. I. Kopelevich, Physica D 63, 299 (1993).

41. M. Abel, H. Chaté and H. Voss, to be published.

42. Another relevant line that appears in the parameter plane is the so-called $L_{2}$-line which is the transition from defect to phase chaos in the hysteretic regime.

43. N. W. Ashcroft and N. D. Mermin, Solid State Physics (Holt, Rinehart and Winston, New York, 1976); P. Collet and J.-P. Eckmann, Instabilities and Fronts in Extended Systems (Princeton University Press, 1990).

44. M. Or-Guil, I. G. Kevrekidis and M. Bär, Physica D 135, 154 (2000). 